AÏSSA WADE

Modèles locaux de structures de Poisson singulières en dimension 3

Bulletin de la S. M. F., tome 125, no 4 (1997), p. 573-618

<http://www.numdam.org/item?id=BSMF_1997_125_4_573_0>

(C) Bulletin de la S. M. F., 1997, tous droits réservés.

L'accès aux archives de la revue «Bulletin de la S. M. F. » (http: //smf.emath.fr/Publications/Bulletin/Presentation.html) implique l'accord avec les conditions générales d'utilisation (http:/www.numdam.org/ conditions). Toute utilisation commerciale ou impression systématique est constitutive d'une infraction pénale. Toute copie ou impression de ce fichier doit contenir la présente mention de copyright.

\title{
Numdam
}

Article numérisé dans le cadre du programme

Numérisation de documents anciens mathématiques

http://www.numdam.org/ 
Bull. Soc. math. France,

125,1997 , p. $573-618$.

\section{MODÈLES LOCAUX DE STRUCTURES DE POISSON \\ SINGULIÈRES EN DIMENSION 3}

PAR

Aïssa WADE $(*)$

RÉSUMÉ. - On étudie la classification des structures de Poisson différentiables de classe $C^{\infty}$ qui ont un 1-jet nul en un point. On montre qu'en dimension 3, dans la famille des structures de Poisson ayant un 2-jet quadratique «diagonalisable», il y a quatre importantes classes. Ensuite, on déduit de cette classification celle des 1-formes différentielles intégrables singulières.

ABSTRACT. - Classification of smooth Poisson structures with zero 1-jet at a point, is studied. In dimension 3 , we show that the family of Poisson structures with quadratic and "diagonalizable» 2-jet, admits four important class. This classification is applied to obtain the classification of singular integrable 1-forms.

\section{Introduction}

Une structure de Poisson sur une variété différentiable $M$ est la donnée sur l'espace $C^{\infty}(M, \mathbb{R})$ des fonctions différentiables, d'une loi de composition interne $\{$,$\} appelée crochet de Poisson, qui en fait une algèbre$ de Lie et qui vérifie l'identité de Leibniz

$$
\{f g, h\}=\{f, h\} g+f\{g, h\},
$$

quels que soient $f, g$ et $h \in C^{\infty}(M, \mathbb{R})$. On dit alors que $(M,\{\}$,$) est$ une variété de Poisson. La donnée d'un crochet de Poisson équivaut à celle d'un champ de tenseurs antisymétriques deux fois contravariants $\Pi$ appelé tenseur de Poisson qui vérifie

$$
[\Pi, \Pi]=0,
$$

(*) Texte reçu le 13 mai 1997, révisé et accepté le 22 octobre 1997.

A. WADE, Département de Mathématiques, c.c. 051, Université Montpellier II, Place Eugène Bataillon, 34095 Montpellier CEDEX 05 (France).

Email : wade@darboux.math.univ-montp2.fr.

Mots clés : Structures de Poisson, singularités, formes intégrables, modèles locaux.

Classification AMS : 58 F 36, 53 B 99, 58 F 14.

BULLETIN DE LA SOCIÉTÉ MATHÉMATIQUE DE FRANCE 0037-9484/1997/573/\$ 5.00

(C) Société mathématique de France 
où le crochet $[$,$] est celui de Schouten. Le tenseur de Poisson s'obtient$ par la relation

$$
\Pi(x)(\mathrm{d} f(x), \mathrm{d} g(x))=\{f, g\}(x) .
$$

On appelle rang de la structure de Poisson en un point $x$, le rang de la forme bilinéaire antisymétrique $\Pi(x)$ sur $T_{x}^{*} M$.

Il est connu que localement, toute variété de Poisson est le produit d'une variété symplectique par une variété de Poisson de rang nul en un point (voir [We1]). Donc, l'étude des formes normales de structures de Poisson n'est intéressante que pour les structures de Poisson de rang nul en un point.

Rappelons le problème des formes normales de structures de Poisson qui peut se formuler comme suit : étant donnés une variété de Poisson $(M,\{\}$,$) et m_{0}$ un point de $M$, on cherche des coordonnées locales $\left(x_{1}, \ldots, x_{n}\right)$ définies au voisinage de $m_{0}$ telles que l'écriture des crochets $\left\{x_{i}, x_{j}\right\}$ soit la plus simple possible.

D'une manière générale, si $\left(x_{1}, \ldots, x_{n}\right)$ sont des coordonnées locales définies sur un ouvert $U$, alors les $\left\{x_{i}, x_{j}\right\}$ déterminent le crochet de Poisson sur $U$. Dans cet ouvert, le tenseur de Poisson s'écrit

$$
\Pi=\sum_{i<j}\left\{x_{i}, x_{j}\right\} \frac{\partial}{\partial x_{i}} \wedge \frac{\partial}{\partial x_{j}}
$$

On s'intéresse ici à l'existence de formes normales polynomiales. En dimension 2 , cette question a été étudiée dans la référence [A], où l'on trouve une liste de modèles locaux de structures de Poisson sur $\mathbb{R}^{2}$. Par ailleurs, le problème qui a été beaucoup étudié, c'est celui de la linéarisation d'une structure de Poisson (en dimension quelconque) de rang nul en un point $m_{0}$. La linéarisation consiste à trouver des coordonnées $\left(x_{1}, \ldots, x_{n}\right)$ nulles en $m_{0}$ dans lesquelles

$$
\left\{x_{i}, x_{j}\right\}=\sum c_{i j}^{k} x_{k}
$$

En fait, les $c_{i j}^{k}$ sont des constantes de structure d'une algèbre de Lie $\mathcal{G}$ intrinsèquement liée au crochet de Poisson qui est appelée linéarisée de la structure de Poisson en $m_{0}$. On montre dans [We1] que lorsque $\mathcal{G}$ est semisimple, la structure de Poisson est formellement linéarisable. Ce résultat a été complété par J. Conn (voir [C1], [C2]) qui montre qu'il reste valable en analytique (en classe $C^{\infty}$ aussi si l'on suppose de plus que $\mathcal{G}$ est de type compacte). La linéarisation est également traitée dans [D], [D-M]. On donne des formes normales formelles en dimension quelconque dans $[\mathrm{H}]$, $[\mathrm{D}-\mathrm{W}]$ et $[\mathrm{Wa} 1]$. 
On connaît peu de chose sur le problème général de l'existence d'une forme normale polynomiale en classe $C^{\infty}$ pour une structure de Poisson de rang nul en un point, exceptés les résultats de linéarisation et ceux de "quadratisation» obtenus dans $[\mathrm{H}]$ et $[\mathrm{D}-\mathrm{W}]$. Il est important de noter que les singularités de tenseur de Poisson avec 1-jet nul sont en général stables en ce sens que de façon générique, si un tenseur de Poisson $\Pi$ admet un 1-jet nul en un point $m_{0}$, alors tout autre tenseur de Poisson qui est $C^{2}$-proche de $\Pi$ possède un 1-jet nul en un point voisin de $m_{0}$.

Les tenseurs de Poisson étudiés ici ont un 1-jet nul en un point; nous étudierons le cas particulier de la dimension 3, cela permet d'obtenir des formes normales polynomiales en classe $C^{\infty}$. Travaillant localement, on supposera que $\left(M, m_{0}\right)=\left(\mathbb{R}^{3}, 0\right)$. Par la contraction avec une forme volume $\Omega$, on associe à tout tenseur de Poisson $\Pi$ une 1-forme différentielle intégrable $\omega=i_{\Pi} \Omega$. Cette correspondance définit un isomorphisme entre l'espace des tenseurs de Poisson sur $\mathbb{R}^{3}$ et celui des 1-formes intégrables. Les singularités des 1-formes différentielles intégrables sur $\mathbb{C}^{n}$ (ou $\mathbb{R}^{n}$ ) ont fait l'objet de nombreuses études notamment celles de C. Camacho, D. Cerveau, R. Moussu, A. Lins Neto, etc.

Si $\omega$ et $\omega^{\prime}$ sont deux 1-formes intégrables conjuguées, c'est-à-dire qu'il existe un difféomorphisme $\varphi$ tel que $\omega^{\prime}=\varphi^{*} \omega$, alors les tenseurs de Poisson $\Pi$ et $\Pi^{\prime}$ associés respectivement à $\omega$ et $\omega^{\prime}$ sont liés par

$$
\Pi^{\prime}=J \varphi \varphi_{*} \Pi,
$$

où $J \varphi$ est le Jacobien de $\varphi$. En d'autres termes, $\Pi$ est isomorphe à $\Pi^{\prime}$, à multiplication par une fonction non nulle près. Mais en général pour se ramener à un modèle local, on se sert de théorèmes généraux qui ne permettent pas d'avoir une expression explicite des isomorphismes $\varphi$, donc on ne maîtrise pas le facteur $J \varphi$. Comme le produit d'un tenseur de Poisson sur $\mathbb{R}^{3}$ par une fonction est toujours un tenseur de Poisson (ceci n'est pas valable en dimension supérieure ou égale à 4 ), on peut dire que la connaissance des modèles locaux des 1-formes intégrables ne donne pas des renseignements sur celle des modèles locaux de tenseurs de Poisson. Par contre, nous montrons dans le paragraphe 5 qu'en partant des résultats sur les tenseurs de Poisson, on trouve aisément les modèles locaux des 1-formes différentielles intégrables.

Nous verrons plus loin que deux 1-formes intégrables conjuguées peuvent correspondre à deux structures de Poisson non isomorphes. En revanche, à chaque modèle de tenseur de Poisson, est associé un unique modèle de 1-forme intégrable.

Dans la classification des structures de Poisson quadratiques en dimension 3 faite dans [D-H], on trouve deux grandes classes. 
La première classe correspond aux formes intégrables exactes, ce sont les structures de Poisson du type

$$
\Pi^{2}=\frac{\partial P}{\partial x_{3}} \frac{\partial}{\partial x_{1}} \wedge \frac{\partial}{\partial x_{2}}+\frac{\partial P}{\partial x_{1}} \frac{\partial}{\partial x_{2}} \wedge \frac{\partial}{\partial x_{3}}+\frac{\partial P}{\partial x_{2}} \frac{\partial}{\partial x_{3}} \wedge \frac{\partial}{\partial x_{1}}
$$

où $P\left(x_{1}, x_{2}, x_{3}\right)$ est un polynôme homogène de degré 3 .

La seconde classe est formée des structures de Poisson isomorphes à une structure de Poisson du type

$$
(* *) \quad \Pi^{2}=c x_{1} x_{2} \frac{\partial}{\partial x_{1}} \wedge \frac{\partial}{\partial x_{2}}+a x_{2} x_{3} \frac{\partial}{\partial x_{2}} \wedge \frac{\partial}{\partial x_{3}}+b x_{3} x_{1} \frac{\partial}{\partial x_{3}} \wedge \frac{\partial}{\partial x_{1}},
$$

avec $a, b$ et $c$ deux à deux distincts. Ces dernières sont dites quadratiques diagonalisables; elles correspondent aux 1-formes intégrables du type

$$
\omega_{2}=a x_{2} x_{3} \mathrm{~d} x_{1}+b x_{1} x_{3} \mathrm{~d} x_{2}+c x_{1} x_{2} \mathrm{~d} x_{3} .
$$

Une telle forme différentielle $\omega_{2}$ est dite tangente à une action commutative linéaire de $\mathbb{R}^{2}$, c'est-à-dire qu'il existe deux champs de vecteurs linéaires commutants $X, Y$ tels que $\left\{X_{x}, Y_{x}\right\}$ soit libre pour tout $x$ élément d'un ouvert dense et tels que

$$
\omega_{2}=i_{X} i_{Y} \mathrm{~d} x_{1} \wedge \mathrm{d} x_{2} \wedge \mathrm{d} x_{3} .
$$

L'intégration des champs de vecteurs de l'algèbre commutative de dimension 2 engendrée par $X$ et $Y$, donne un groupe $G$ de germes de difféomorphismes qui agit naturellement sur $\left(\mathbb{R}^{3}, 0\right)$.

D'après [Ce-LN], toute 1-forme intégrable sur $\mathbb{R}^{3}$ dont le 2-jet à l'origine est égal à $\omega_{2}$ (avec $a, b, c$ non nuls et deux à deux distincts), est tangente à une action commutative. Nous précisons ce résultat en montrant que les modèles locaux de telles 1-formes sont presque toujours tangentes à une action commutative polynomiale de $\mathbb{R}^{2}$ que l'on sait déterminer. Nous prouvons d'abord le théorème qui suit.

ThÉORÈme. - Soit $\Pi$ une structure de Poisson sur $\mathbb{R}^{3}$ dont le jet d'ordre 1 est nul à l'origine. On suppose qu'il existe des coordonnées $\left(x_{1}, x_{2}, x_{3}\right)$ nulles en 0 telles le 2 -jet de $\Pi$ en 0 s'écrit sous la forme $\left.{ }^{* *}\right)$, les coefficients $a, b$ et $c$ étant deux à deux distincts. On suppose également que si l'un des réels $a, b$ ou $c$ est nul, les deux autres sont de signe contraire. Alors $\Pi$ est isomorphe en classe $C^{\infty}$ à l'un des modèles locaux suivants

$$
\begin{aligned}
& M_{0}=c x y \frac{\partial}{\partial x} \wedge \frac{\partial}{\partial y}+a y z \frac{\partial}{\partial y} \wedge \frac{\partial}{\partial z}+b z x \frac{\partial}{\partial z} \wedge \frac{\partial}{\partial x} \\
& M_{1}=M_{0}+y^{m_{1}} z^{m_{2}} \frac{\partial}{\partial x} \wedge\left(\alpha y \frac{\partial}{\partial y}+z \frac{\partial}{\partial z}\right)
\end{aligned}
$$

TOME $125-1997-\mathrm{N}^{\circ} 4$ 
avec $\left(m_{1}, m_{2}\right) \in \mathbb{N}^{2}, m_{1}+m_{2} \geq 2$ et $\alpha \in \mathbb{R} ;$

$$
M_{2}=\left(1 \pm \varrho^{p}\right) M_{0}
$$

où $\varrho$ est un polynôme homogène en $x, y, z$ et $p \in \mathbb{N}^{*}$;

$$
M_{3}=P_{q-1}(\varrho) M_{0}+\varrho^{q} \widetilde{\Pi}_{2},
$$

où $P_{q-1}$ est un polynôme en $\varrho$ de degré $(q-1)$, avec $q \in \mathbb{N}^{*}$ et $\widetilde{\Pi}_{2}$ est un tenseur de Poisson d'ordre 2 en 0.

En se servant de ce résutat, nous établissons le théorème :

THÉORÈME. - Soit $\omega$ une 1-forme intégrable de classe $C^{\infty}$ au voisinage de l'origine $0 \in \mathbb{R}^{3}$ ayant pour 2-jet en ce point

$$
\omega_{2}=a y z \mathrm{~d} x+b z x \mathrm{~d} y+c x y \mathrm{~d} z
$$

avec $(a-b)(b-c)(c-a) \neq 0$. Supposons que si l'un des coefficients $a, b$ ou $c$ est nul, les deux autres sont de signe contraire. Alors $\omega$ est $C^{\infty}$-conjuguée à l'un des modèles suivants :

$$
\begin{aligned}
& \mathcal{F}_{0}=\omega_{2}, \\
& \mathcal{F}_{1}=\omega_{2}+y^{m_{1}} z^{m_{2}}(\alpha y \mathrm{~d} z-z \mathrm{~d} y), \\
& \mathcal{F}_{2}=\omega_{2}+\varrho^{q} \widetilde{\omega}_{2},
\end{aligned}
$$

où $\varrho$ est un polynôme homogène en $x, y, z$ et $\widetilde{\omega}_{2}=\tilde{a} y z d x+\tilde{b} z x d y+\tilde{c} x y d z$, le triplet $(\tilde{a}, \tilde{b}, \tilde{c})$ n'étant pas colinéaire à $(a, b, c)$.

Ce travail comporte cinq paragraphes. Dans le premier, nous rappelons quelques définitions de base. Les paragraphes 2,3 et 4 sont consacrés à la démonstration du premier théorème. Dans le dernier paragraphe, nous montrons le second théorème.

\section{Rappels sur les structures de Poisson}

Considérons un crochet de Poisson sur une variété $M$, c'est-à-dire une application bilinéaire, antisymétrique

$$
\{,\}: C^{\infty}(M, \mathbb{R}) \times C^{\infty}(M, \mathbb{R}) \longrightarrow C^{\infty}(M, \mathbb{R})
$$

qui vérifient les identités de Jacobi et Leibniz

$$
\begin{gathered}
\{f,\{g, h\}\}+\{g,\{h, f\}\}+\{h,\{f, g\}\}=0, \\
\{f g, h\}=\{f, h\} g+f\{g, h\},
\end{gathered}
$$

quels que soient $f, g$ et $h \in C^{\infty}(M, \mathbb{R})$. 
À ce crochet de Poisson, il correspond un unique champ de tenseurs antisymétriques deux fois contravariants $\Pi$ défini par

$$
\Pi(\mathrm{d} f, \mathrm{~d} g)=\{f, g\},
$$

il est appelé tenseur de Poisson. Plus précisément, la donnée d'un crochet de Poisson sur $M$ équivaut à celle d'un champ de tenseurs antisymétriques deux fois contravariants $\Pi$ qui satisfait la relation

$$
[\Pi, \Pi]=0,
$$

où le crochet $[$,$] est celui de Schouten. Cette relation permet d'avoir$ l'identité de Jacobi.

Il résulte de l'identité de Leibniz que pour toute fonction différentiable $h$ fixée, l'application

$$
\operatorname{ad}_{h}=\{., h\}
$$

est une dérivation de la structure d'algèbre associative définie par le produit usuel des fonctions différentiables : il existe un unique champ de vecteurs différentiable $X_{h}$ sur $M$ tel que $\operatorname{ad}_{h}=X_{h}$.

On dit que $X_{h}$ est le champ de vecteurs hamiltonien de $h$. Lorsque $X_{h}$ est nul, on dit que $h$ est une fonction de Casimir pour la structure de Poisson.

L'ensemble des champs de vecteurs hamiltoniens est un espace vectoriel réel stable par le crochet de Lie. On a l'égalité :

$$
\left[X_{f}, X_{g}\right]=X_{\{f, g\}}
$$

pour tous $f$ et $g \in C^{\infty}(M)$. Toute structure de Poisson donne lieu à un feuilletage singulier ou «de Stefan» qui est engendré par les champs de vecteurs hamiltoniens et dont chacune des feuilles est munie d'une structure de variété symplectique.

DÉfinition. - Une application $\varphi:\left(M_{1}, \Pi_{1}\right) \rightarrow\left(M_{2}, \Pi_{2}\right)$ entre deux variétés de Poisson, est appelée morphisme de Poisson si quels que soient $f, g \in C^{\infty}\left(M_{2}, \mathbb{R}\right)$ on a la relation

$$
\{f, g\}_{2} \circ \varphi=\{f \circ \varphi, g \circ \varphi\}_{1},
$$

où $\{,\}_{1}$ et $\{,\}_{2}$ sont respectivement les crochets de Poisson associés à $\Pi_{1}$ et $\Pi_{2}$. Cela revient à dire qu'on a la relation

$$
\varphi_{*} \Pi_{1}=\Pi_{2} \text {. }
$$

Si de plus, $\varphi$ est un difféomorphisme de classe $C^{\infty}$ (resp. analytique, TOME $125-1997-\mathrm{N}^{\circ} 4$ 
formel), on dit que $\varphi$ est un isomorphisme de Poisson de classe $C^{\infty}$ (resp. analytique, formel).

Définition. - On dira qu'une structure de Poisson $\Pi$ différentiable (resp. analytique, formelle) est diagonale dans les coordonnées $\left(x_{1}, \ldots, x_{n}\right)$ si elle s'écrit

$$
\Pi=\sum_{i<j} \bar{\Pi}_{i j} x_{i} x_{j} \frac{\partial}{\partial x_{i}} \wedge \frac{\partial}{\partial x_{j}},
$$

les $\bar{\Pi}_{i j}$ étant des fonctions différentiables (resp. des fonctions analytiques, des séries formelles).

\section{Modèles locaux formels}

Fixons d'abord les notations que nous utiliserons par la suite.

Notations. - Soient $\left(x_{1}, x_{2}, x_{3}\right)$ des coordonnées locales autour de 0. Si $f$ est une série formelle, nous écrivons :

$$
f=\sum_{I \in \mathbb{N}^{3}} f^{I} x^{I} \quad \text { avec } \quad x^{I}=x_{1}^{I_{1}} x_{2}^{I_{2}} x_{3}^{I_{3}} \quad \text { et } \quad f^{I} \in \mathbb{R} .
$$

Un champ de vecteurs formel $X$ s'écrit :

$$
X=\sum_{i=1}^{3} \sum_{I \in \mathbb{N}^{3}} a_{i}^{I} x^{I} \frac{\partial}{\partial x_{i}} \quad \text { avec } \quad a_{i}^{I} \in \mathbb{R}
$$

Posons $x^{J}=x^{I} / x_{i}$, c'est-à-dire que $J_{\ell}=I_{\ell}$, pour tout $\ell$ différent de $i$ et $J_{i}=I_{i}-1$. Nous obtenons l'écriture

$$
X=\sum_{i=1}^{3} \sum_{J} \eta_{i}^{J} x^{J} x_{i} \frac{\partial}{\partial x_{i}}
$$

Les triplets $J$ sont dans $(\mathbb{N} \cup\{-1\})^{3}$; ils possèdent au plus une composante égale à -1 . Nous conviendrons que si l'une des composantes $J_{r}$ est égale à -1 , alors $\eta_{i}^{J}$ est nul pour tout $i$ différent de $r$.

Un champ de bivecteurs formel $P$ s'écrit

$$
P=\sum_{i<j} \sum_{I \in \mathbb{N}^{3}} P_{i j}^{I} x^{I} \frac{\partial}{\partial x_{i}} \wedge \frac{\partial}{\partial x_{j}} \quad \text { avec } \quad P_{i j}^{I} \in \mathbb{R} .
$$


Remplaçons $x^{I}$ par $x^{K} / x_{i} x_{j}$, avec $K_{r}=I_{r}$ pour tout $r$ différent de $i$ et $j$, $K_{i}=I_{i}-1$ et $K_{j}=I_{j}-1$. Il vient :

$$
P=\sum_{i<j} \sum_{K} \alpha_{i j}^{K} x^{K} x_{i} x_{j} \frac{\partial}{\partial x_{i}} \wedge \frac{\partial}{\partial x_{j}} .
$$

Les triplets $K$ sont dans $(\mathbb{N} \cup\{-1\})^{3}$, ils contiennent au plus deux composantes égales à -1 . De plus,

- si $K$ contient une composante négative $K_{k}=-1$ alors $\alpha_{i j}^{K}$ est nul à chaque fois que $i$ et $j$ sont différents de $k$;

- si $K$ contient deux composantes négatives $K_{r}=K_{s}=-1$, les $\alpha_{i j}^{K}$ sont tous nuls sauf peut-être pour $\{i, j\}=\{r, s\}$.

Dans la suite, nous emploierons surtout les notations (1) et (2), elles permettent d'allèger les calculs. Pour obtenir les modèles locaux formels, nous utilisons un résultat de forme normale formelle valable en toutes dimensions qui a été établi dans $[\mathrm{D}-\mathrm{W}]$. En dimension 3 , il peut s'énoncer comme suit.

Proposition 2.1. - Soit $\Pi$ une structure de Poisson sur $\mathbb{R}^{3}$. On suppose qu'il existe des coordonnées $\left(x_{1}, x_{2}, x_{3}\right)$ nulles en 0 dans lesquelles le 2-jet de П en 0 s'écrit

$$
\Pi^{2}(x)=c x_{1} x_{2} \frac{\partial}{\partial x_{1}} \wedge \frac{\partial}{\partial x_{2}}+a x_{2} x_{3} \frac{\partial}{\partial x_{2}} \wedge \frac{\partial}{\partial x_{3}}+b x_{3} x_{1} \frac{\partial}{\partial x_{3}} \wedge \frac{\partial}{\partial x_{1}}
$$

où les réels $a, b$ et $c$ sont deux à deux distincts. Alors il existe un changement de coordonnées formel $\varphi:\left(x_{1}, x_{2}, x_{3}\right) \mapsto\left(y_{1}, y_{2}, y_{3}\right)$ vérifiant

$$
\varphi_{*} \Pi=\Pi^{2}(y)+\sum_{J \|(a, b, c)} \alpha_{i j}^{J} y^{J} y_{i} y_{j} \frac{\partial}{\partial y_{i}} \wedge \frac{\partial}{\partial y_{j}}
$$

La notation $J \|(a, b, c)$ signifie que $J$ est colinéaire à $(a, b, c)$.

Preuve. - Nous construisons par récurrence des coordonnées formelles qui satisfont la proposition. Supposons que $\left(x_{1}, x_{2}, x_{3}\right)$ sont des coordonnées nulles en 0 telles qu'on ait :

$$
\left\{x_{i}, x_{j}\right\}=\sum_{\ell \geq 2} \Pi_{i j}^{\ell}=a_{i j} x_{i} x_{j}+\sum_{\substack{J \|(a, b, c) \\ 1 \leq|J| \leq p-2}} \alpha_{i j}^{J} x^{J} x_{i} x_{j}+\Pi_{i j}^{p+1}+\cdots,
$$

où $\Pi_{i j}^{\ell}$ désigne le terme homogène de degré $\ell, a=a_{23}, b=a_{31}$ et $c=a_{12}$.

$$
\text { TOME } 125-1997-\mathrm{N}^{\circ} 4
$$


Remarquons d'abord que pour tout $\ell \leq p$ et pour tout $i$ différent de $j$, le terme $\Pi_{i j}^{\ell}$ ne contient pas de monôme du type $x_{k}^{\ell}$, avec $k \neq i, j$. Puisque, sinon on aurait un triplet non nul $J$ colinéaire à $(a, b, c)$ avec $J_{i}=J_{j}=-1$; ce qui contredit le fait que $a, b$ et $c$ sont deux à deux distincts. En utilisant l'identité de Jacobi, nous montrons que $\Pi_{i j}^{p+1}$ ne comporte pas non plus de tels monômes. En effet, nous pouvons écrire

$$
\Pi_{i j}^{p+1}=\sum_{|J|=p-1} \alpha_{i j}^{J} x^{J} x_{i} x_{j},
$$

où les $x^{J} x_{i} x_{j}$ sont homogènes de degré $p+1$, avec $p \geq 2$.

Dire que $\Pi_{i j}^{p+1}$ comporte un monôme non nul en $x_{k}^{p+1}$ (avec $k \neq i, j$ ) revient à dire que $\alpha_{i j}^{J}$ est non nul si $J_{i}=J_{j}=-1$ et $J_{k}=p+1$. Or, en considérant uniquement les termes en $x_{k}^{p+2}$ dans l'identité de Jacobi

$$
\left\{\left\{x_{i}, x_{j}\right\}, x_{k}\right\}+\left\{\left\{x_{j}, x_{k}\right\}, x_{i}\right\}+\left\{\left\{x_{k}, x_{i}\right\}, x_{j}\right\}=0
$$

où $\{i, j, k\}=\{1,2,3\}$, nous obtenons

$$
\left(a_{j k}+a_{i k}\right) \alpha_{i j}^{J} x_{k}^{p+2}=0 .
$$

Comme $\{a, b, c\}=\left\{a_{i j}, a_{j k}, a_{k i}\right\}$, et les réels $a, b$ et $c$ sont deux à deux distincts, donc on a nécessairement $\alpha_{i j}^{J}=0$. Par conséquent, $\Pi_{i j}^{p+1}$ ne contient pas de tels monômes.

Maintenant, fixons un triplet $J=I$ qui intervient dans l'écriture de $\Pi_{i j}^{p+1}$ et qui n'est pas colinéaire à $(a, b, c)$. Nous allons montrer qu'il existe un changement de coordonnées $\psi:\left(x_{1}, x_{2}, x_{3}\right) \mapsto\left(z_{1}, z_{2}, z_{3}\right)$ qui élimine le terme en $x^{I} x_{i} x_{j}$, c'est-à-dire qu'on a

$$
\begin{aligned}
& \left\{z_{r}, z_{s}\right\}=a_{r s} z_{r} z_{s}+\sum_{\substack{J \|(a, b, c) \\
1 \leq|J| \leq p-2}} \alpha_{r s}^{J} z^{J} z_{r} z_{s} \\
& +\sum_{\substack{J|=p-1\\
| J \neq s \\
J \neq I}} \beta_{r s}^{J} z^{J} z_{r} z_{s}+\Pi_{i j}^{p+2}+\cdots
\end{aligned}
$$

Premier cas : supposons que $I$ est dans $\mathbb{N}^{3}$. Pour tout $r=1, \ldots, n$, posons :

$$
z_{r}=x_{r} \theta_{r} \quad \text { avec } \theta_{r}=1+\varphi_{r} x^{I}, \varphi_{r} \in \mathbb{R} .
$$

Il s'agit donc de déterminer les réels $\varphi_{r}$ de façon que (3) soit vérifié. Nous avons

$$
\left\{z_{r}, z_{s}\right\}=\theta_{r} \theta_{s}\left\{x_{r}, x_{s}\right\}+x_{s} \theta_{r}\left\{x_{r}, \theta_{s}\right\}+x_{r} \theta_{s}\left\{\theta_{r}, x_{s}\right\}
$$


ou encore

$$
\left\{z_{r}, z_{s}\right\}=\theta_{r} \theta_{s}\left\{x_{r}, x_{s}\right\}+x_{s} \theta_{r} \sum_{\ell} \frac{\partial \theta_{s}}{\partial x_{\ell}}\left\{x_{r}, x_{\ell}\right\}-x_{r} \theta_{s} \sum_{\ell} \frac{\partial \theta_{r}}{\partial x_{\ell}}\left\{x_{s}, x_{\ell}\right\} .
$$

En posant

$$
\Lambda_{r s}(x)=\sum_{\substack{1 \leq|J| \leq p-1 \\ J \neq I}} x^{J} \alpha_{r s}^{J} \quad \text { et } \quad A_{r} \cdot I=\sum_{s} a_{r s} I_{s}
$$

nous pouvons écrire

$$
\left\{z_{r}, z_{s}\right\}=\theta_{r} \theta_{s} x_{r} x_{s}\left(a_{r s}+\Lambda_{r s}(x)+x^{I}\left(\alpha_{r s}^{I}+A_{r} \cdot I \varphi_{s}-A_{s} \cdot I \varphi_{r}\right)\right)+O\left(\|x\|^{p+1}\right) .
$$

Si le système d'équations qui suit

$$
\alpha_{r s}^{I}=A_{s} \cdot I \varphi_{r}-A_{r} \cdot I \varphi_{s}
$$

admet une solution alors compte tenu du fait que le changement de coordonnées réciproque de $\varphi$ est donnée par

$$
x_{r}=z_{r}\left(1-\varphi_{r}^{I} z^{I}\right)+0\left(\|z\|^{p-1}\right)
$$

nous obtenons la relation (3). Il suffit donc de montrer qu'il existe des $\varphi_{r}$ qui vérifient (4). Or, avec les notations

$$
\begin{gathered}
A \cdot I=\sum_{r} A_{r} \cdot I x_{r} \frac{\partial}{\partial x_{r}} \\
\Pi^{I}=\sum_{r<s} \alpha_{r s}^{I} x_{s} x_{r} \frac{\partial}{\partial x_{r}} \wedge \frac{\partial}{\partial x_{s}} \\
Z=\sum_{r} \varphi_{r} x_{r} \frac{\partial}{\partial x_{r}}
\end{gathered}
$$

les équations (4) deviennent

$$
\Pi^{I}=Z \wedge A \cdot I
$$

Mais, $A \cdot I \wedge \Pi^{I}=0$ (à cause de l'identité de Jacobi) et $A \cdot I$ est non nul car sinon $I$ serait colinéaire à $(a, b, c)$; donc il existe un champ de vecteurs $Z$ qui satisfait (5). Par conséquent, si $I$ est dans $\mathbb{N}^{3}$, on peut trouver un changement de coordonnées qui satisfait (3).

TOME $125-1997-\mathrm{N}^{\circ} 4$ 
Deuxième cas : supposons que $I_{i}=-1$, les autres composantes de $I$ étant dans $\mathbb{N}$. - Posons

$$
\begin{array}{ll}
z_{r}=x_{r} & \text { pour } r \neq i, \\
z_{i}=x_{i} \theta_{i}, & \text { avec } \theta_{i}=1+\varphi_{i} x^{I} .
\end{array}
$$

Des calculs identiques à ceux faits dans le premier cas montrent que l'on peut trouver $\varphi_{i}$ tel que (3) soit réalisé.

Par itération, on élimine au moyen d'un changement de coordonnées tous les termes homogènes de degré $p+1$ correspondant à un triplet $I$ qui n'est pas colinéaire à $(a, b, c)$. Ainsi, on aboutit au résultat souhaité de proche en proche.

Considérons la structure de Poisson formelle

$$
\varphi_{*} \Pi=\Pi^{2}(y)+\sum_{J \|(a, b, c)} \alpha_{i j}^{J} y^{J} y_{i} y_{j} \frac{\partial}{\partial y_{i}} \wedge \frac{\partial}{\partial y_{j}} .
$$

De deux choses l'une :

- Ou bien $(a, b, c)$ n'est colinéaire à aucun triplet de $(\mathbb{N} \cup\{-1\})^{3} ;$ dans ce cas $\varphi_{*} \Pi=\Pi^{2}$. Donc, $\Pi$ est formellement isomorphe à son 2-jet en 0 .

- Ou bien $(a, b, c)$ est colinéaire à un triplet de $(\mathbb{N} \cup\{-1\})^{3}$. Comme tout multiple réel de $\Pi^{2}$ (en particulier son opposé) possède les mêmes propriétés que $\Pi^{2}$, on peut restreindre l'étude aux deux cas suivants : $a<0 \leq b<c$ et $0 \leq a<b<c$.

(i) Supposons que $a<0 \leq b<c$; on a :

$$
(a, b, c)=-a\left(-1,-\frac{b}{a},-\frac{c}{a}\right)=-a\left(-1, m_{1}, m_{2}\right) .
$$

Le vecteur $J=\left(-1, m_{1}, m_{2}\right)$ est l'unique triplet de $(\mathbb{N} \cup\{-1\})^{3}$ qui soit colinéaire à $(a, b, c)$. On en déduit qu'il existe deux constantes $\alpha$ et $\beta$ telles que l'on ait formellement

$$
\varphi_{*} \Pi=\Pi^{2}(y)+y_{2}^{m_{1}} y_{3}^{m_{2}} \frac{\partial}{\partial y_{1}} \wedge\left(\alpha y_{2} \frac{\partial}{\partial y_{2}}+\beta y_{3} \frac{\partial}{\partial y_{3}}\right) .
$$

Nous verrons plus loin que l'on peut supposer que $\beta=1$.

(ii) Supposons maintenant que $0 \leq a<b<c$. Alors, les $J$ colinéaires au vecteur $(a, b, c)$ sont dans $\mathbb{N}^{3}$. 
Notations. - Désignons par $I_{0}=\left(n_{1}, n_{2}, n_{3}\right)$ le plus petit triplet de $\mathbb{N}^{3}$ qui soit colinéaire à $(a, b, c)$, en ce sens que tout autre triplet de $\mathbb{N}^{3}$ colinéaire à $(a, b, c)$ est un multiple entier de $I_{0}$. Notons

$$
\varrho\left(y_{1}, y_{2}, y_{3}\right)=y_{1}^{n_{1}} y_{2}^{n_{2}} y_{3}^{n_{3}} \text {. }
$$

Nous obtenons

$$
\varphi_{*} \Pi=\sum_{i<j} f_{i j}(\varrho) y_{i} y_{j} \frac{\partial}{\partial y_{i}} \wedge \frac{\partial}{\partial y_{j}} .
$$

Nous avons la classification formelle suivante.

THÉORÈme 2.1. $-S i(a-b)(b-c)(c-a) \neq 0$ alors toute structure de Poisson sur $\mathbb{R}^{3}$, ayant $\Pi^{2}$ pour 2-jet à l'origine, est formellement isomorphe à l'un des modèles suivants

$$
\begin{aligned}
& M_{0}=c x y \frac{\partial}{\partial x} \wedge \frac{\partial}{\partial y}+a y z \frac{\partial}{\partial y} \wedge \frac{\partial}{\partial z}+b z x \frac{\partial}{\partial z} \wedge \frac{\partial}{\partial x}, \\
& M_{1}=M_{0}+y^{m_{1}} z^{m_{2}} \frac{\partial}{\partial x} \wedge\left(\alpha y \frac{\partial}{\partial y}+z \frac{\partial}{\partial z}\right),
\end{aligned}
$$

$\operatorname{avec}\left(m_{1}, m_{2}\right) \in \mathbb{N}^{2}, m_{1}+m_{2} \geq 2$ et $\alpha \in \mathbb{R}$,

$$
M_{2}=\left(1 \pm \varrho^{p}\right) M_{0},
$$

où $\varrho$ est un polynôme homogène en $x, y, z$ et $p \in \mathbb{N}^{*}$,

$$
M_{3}=\left(1+\alpha_{1} \varrho+\cdots+\alpha_{q-1} \varrho^{q-1}\right) \Pi^{2}+\varrho^{q} \widetilde{\Pi}^{2}
$$

où les $\alpha_{i}$ sont des réels et $\widetilde{\Pi}^{2}$ est un tenseur de Poisson d'ordre 2 en 0 qui n'est pas un multiple de $\Pi^{2}$.

En vertu du raisonnement tenu plus haut, pour prouver ce théorème, il suffit de montrer que la structure de Poisson donnée par (6) est formellement isomorphe à l'un des modèles $M_{2}$ ou $M_{3}$. Pour cela, nous emploierons les quatre lemmes qui suivent (ils sont valables en toutes dimensions).

Soit $I_{0}=\left(n_{1}, \ldots, n_{m}\right) \in \mathbb{N}^{m}$ fixé. On pose

$$
\varrho\left(y_{1}, \ldots, y_{m}\right)=y_{1}^{n_{1}} \times \cdots \times y_{m}^{n_{m}} .
$$

TOME $125-1997-\mathrm{N}^{\circ} 4$ 
La structure de Poisson formelle considérée est définie sur $\mathbb{R}^{m}$ par :

$$
\Pi^{\prime}=\sum_{i<j} f_{i j}(\varrho) y_{i} y_{j} \frac{\partial}{\partial y_{i}} \wedge \frac{\partial}{\partial y_{j}} .
$$

On désigne par $F(\varrho)$, la matrice antisymétrique de coefficients $f_{i j}(\varrho)$ et on note

$$
F_{i}(\varrho) \cdot I_{0}=\sum_{j=1}^{m} f_{i j}(\varrho) n_{j} .
$$

Alors, le produit de cette matrice par $I_{0}$ est donnée par :

$$
F(\varrho) \cdot I_{0}=\left(F_{1}(\varrho) \cdot I_{0}, \ldots, F_{m}(\varrho) \cdot I_{0}\right) .
$$

Par la suite, pour simplifier l'écriture, on notera $F_{i}$ au lieu de $F_{i}(\varrho) \cdot I_{0}$.

Remarque. - Les fonctions $F_{i}$ déterminent le champ de vecteurs hamiltonien de la fonction $\varrho$. En effet, on a :

$$
X_{\varrho}=\sum_{1 \leq i, \ell \leq m} \frac{\partial \varrho}{\partial y_{\ell}}\left\{y_{i}, y_{\ell}\right\} \frac{\partial}{\partial y_{i}}=\varrho \sum_{1 \leq i, \ell \leq m} f_{i \ell}(\varrho) n_{\ell} y_{i} \frac{\partial}{\partial y_{i}}=\varrho \sum_{i=1}^{m} F_{i} y_{i} \frac{\partial}{\partial y_{i}} .
$$

Lemme 2.1. - On a les relations

$$
f_{i j}^{\prime} F_{k}+f_{j k}^{\prime} F_{i}+f_{k i}^{\prime} F_{j}=0 .
$$

Preuve. - Il suffit d'écrire l'identité de Jacobi $\left[\varphi_{*} \Pi, \varphi_{*} \Pi\right]=0$ en partant de l'écriture (6) et en utilisant la relation

$$
\begin{aligned}
& {[X \wedge Y, Z \wedge T]=[X, Z] \wedge Y \wedge T-[X, T] \wedge Y \wedge Z } \\
&-[Y, Z] \wedge X \wedge T+[Y, T] \wedge X \wedge Z
\end{aligned}
$$

quels que soient les champs de vecteurs $X, Y, Z$ et $T$.

Lemme 2.2. - Il existe un entier positif $q$, des réels $\mu_{1}, \ldots, \mu_{m}$ et une fonction $G$ tels que

$$
F_{i}=\mu_{i} \varrho^{q} G \quad \text { avec } \quad G(0)=1 .
$$

Preuve. - Lorsque les $F_{i}$ sont toutes nulles (c'est-à-dire que $\varrho$ est une fonction de Casimir pour $\Pi^{\prime}$ ), il suffit de prendre les $\mu_{i}$ tous nuls; alors n'importe quelle fonction $G$ telle que $G(0)=1$ convient. 
Supposons que les fonctions $F_{i}$ ne sont pas toutes nulles au voisinage de zéro. Multiplions (7) par $n_{k}$ et faisons la somme terme à terme lorsque $k$ varie de 1 à $m$. Comme, la somme des $F_{k} n_{k}$ est nulle (à cause de l'antisymétrie de la matrice des $f_{i j}$ ), on obtient :

$$
F_{j}^{\prime} F_{i}-F_{i}^{\prime} F_{j}=0 .
$$

Il existe un indice $i$ et un entier $\ell$ tels que la dérivée d'ordre $\ell$ de $F_{i}$ ne s'annule pas en zéro. Soit $q$ le plus petit de tels entiers. Posons $F_{i}=\varrho^{q} G_{i}$; alors le système (8) donne

$$
G_{j}^{\prime} G_{i}-G_{i}^{\prime} G_{j}=0 .
$$

Quitte à changer l'ordre des indices, on peut toujours supposer que la dérivée d'ordre $q$ de $F_{1}$ en zéro est non nulle. Des relations (9), il résulte alors que les fonctions $G_{i} / G_{1}$ sont constantes. Il suffit donc de prendre

$$
\mu_{i}=\frac{G_{i}}{G_{1}} G_{1}(0) \quad \text { et } \quad G=\frac{G_{1}}{G_{1}(0)} .
$$

Ce qui achève la démonstration.

LEMme 2.3. - Il existe des constantes $c_{i j k}$ telles que

$$
f_{i j} \mu_{k}+f_{j k} \mu_{i}+f_{k i} \mu_{j}=c_{i j k} .
$$

Preuve. - En remplaçant dans les relations (7) du lemme 2.1, $F_{\ell}$ par $\mu_{\ell} \varrho^{\ell} G$ et en tenant compte du fait que $G$ ne s'annule pas au voisinage de zéro, nous obtenons

$$
f_{i j}^{\prime} \mu_{k}+f_{j k}^{\prime} \mu_{i}+f_{k i}^{\prime} \mu_{j}=0
$$

Ce qui signifie que les fonctions $f_{i j} \mu_{k}+f_{j k} \mu_{i}+f_{k i} \mu_{j}$ sont constantes. D'où le lemme.

Notation. - Pour toute fonction $H$ définie sur $\mathbb{R}$ et pour tout $p \in \mathbb{N}^{*}$, on note $H^{[p]}$ le jet d'ordre $p$ de $H$ en zéro.

Lemme 2.4. - Si @ n'est pas une fonction de Casimir pour la structure de Poisson $\Pi^{\prime}$ alors il existe $m$ fonctions $\theta_{1}(\varrho), \ldots, \theta_{m}(\varrho)$ telles qu'on ait :

$$
\left\{y_{i} \theta_{i}, y_{j} \theta_{j}\right\}=y_{i} y_{j} \theta_{i} \theta_{j} f_{i j}^{[q]}(\varrho) \quad \text { avec } \quad \theta_{i}(0)=1 .
$$

TOME $125-1997-\mathrm{N}^{\circ} 4$ 
On rappelle que l'entier $q$ est donné par le lemme 2.2.

Preuve. - On a :

$$
\left\{y_{i} \theta_{i}, y_{j} \theta_{j}\right\}=\theta_{i} \theta_{j}\left\{y_{i}, y_{j}\right\}+\theta_{i} y_{j}\left\{y_{i}, \theta_{j}\right\}+\theta_{j} y_{i}\left\{\theta_{i}, y_{j}\right\}
$$

ou encore

$$
\left\{y_{i} \theta_{i}, y_{j} \theta_{j}\right\}=y_{i} y_{j} \theta_{i} \theta_{j}\left(f_{i j}+\varrho\left(\frac{\theta_{j}^{\prime}}{\theta_{j}} F_{i}-\frac{\theta_{i}^{\prime}}{\theta_{i}} F_{j}\right)\right) .
$$

En vertu du lemme 2.2, il vient :

$$
\left\{y_{i} \theta_{i}, y_{j} \theta_{j}\right\}=y_{i} y_{j} \theta_{i} \theta_{j}\left(f_{i j}+\varrho^{q+1} G\left(\frac{\theta_{j}^{\prime}}{\theta_{j}} \mu_{i}-\frac{\theta_{i}^{\prime}}{\theta_{i}} \mu_{j}\right)\right) .
$$

Donc les fonctions $\theta_{i}$ cherchées doivent vérifier le système

$$
f_{i j}-f_{i j}^{[q]}=\varrho^{q+1} G\left(\frac{\theta_{i}^{\prime}}{\theta_{i}} \mu_{j}-\frac{\theta_{j}^{\prime}}{\theta_{j}} \mu_{i}\right) .
$$

Or, le lemme 2.3 permet d'écrire

$$
\left(f_{i j}-f_{i j}^{[q]}\right) \mu_{k}+\left(f_{j k}-f_{j k}^{[q]}\right) \mu_{i}+\left(f_{k i}-f_{k i}^{[q]}\right) \mu_{j}=0 .
$$

Donc, le système (10) est résoluble. Il suffit de prendre

$$
\frac{\theta_{i}^{\prime}}{\theta_{i}}=-\frac{f_{1 i}-f_{1 i}^{[q]}}{\mu_{1} \varrho^{q+1} G}
$$

D'où le lemme.

Revenons dans le cas de la dimension 3, on a la proposition suivante :

Proposition 2.2. - Il existe un changement de coordonnées

$$
\psi:\left(y_{1}, y_{2}, y_{3}\right) \longmapsto\left(z_{1}, z_{2}, z_{3}\right)
$$

fixant 0 et vérifiant la relation :

$$
\psi_{*} \Pi^{\prime}=f(\varrho) \Pi^{2}+h(\varrho) \bar{\Pi}_{2},
$$

où $f$ est une fonction qui vaut 1 en zéro, $h$ une fonction dont le $(q-1)$-jet est nul en zéro et

$$
\bar{\Pi}_{2}=\frac{1}{n_{3}}\left(\mu_{2} z_{2} z_{2} \frac{\partial}{\partial z_{2}} \wedge \frac{\partial}{\partial z_{2}}-\mu_{1} z_{3} z_{1} \frac{\partial}{\partial z_{3}} \wedge \frac{\partial}{\partial z_{1}}\right)
$$

BULLETIN DE LA SOCIÉTÉ MATHÉMATIQUE DE FRANCE 
Preuve. - Le résultat est immédiat si les fonctions $F_{i}$ sont nulles. En effet, dire qu'elles sont nulles équivaut à dire que le produit de la matrice antisymétrique $\left(f_{i j}\right)$ par $I_{0}$ est nul. En d'autres termes, le produit vectoriel de $I_{0}$ par le vecteur $\left(f_{23}(\varrho), f_{31}(\varrho), f_{12}(\varrho)\right)$ est nul. Par conséquent, ce dernier est soit nul, soit colinéaire à $I_{0}$. Il existe une fonction $\widetilde{f}(\varrho)$ telle qu'on ait :

$$
\left(f_{23}(\varrho), f_{31}(\varrho), f_{12}(\varrho)\right)=\tilde{f}(\varrho) I_{0} .
$$

Or, $I_{0}$ est colinéaire à $(a, b, c)$; donc il existe un réel non nul $\lambda$ tel que $I_{0}=\lambda(a, b, c)$. En posant $f=\lambda \tilde{f}(\varrho)$, il vient

$$
\left(f_{23}(\varrho), f_{31}(\varrho), f_{12}(\varrho)\right)=f(\varrho)(a, b, c)
$$

Pour $\varrho=0$ on a :

$$
(a, b, c)=\left(f_{23}(0), f_{31}(0), f_{12}(0)\right)=f(0)(a, b, c) .
$$

D'où $f(0)=1$. Il suffit donc de prendre $\varphi=\operatorname{id}_{\mathbb{R}^{3}}$ et $h=0$.

Si les $F_{i}$ ne sont pas toutes nulles, on se sert du lemme 2.4 qui assure l'existence de trois fonctions $\theta_{1}(\varrho), \theta_{2}(\varrho)$ et $\theta_{3}(\varrho)$ telles que $\theta_{i}(0)=1$, pour $i \in\{1,2,3\}$ et satisfaisant les relations :

$$
\left\{y_{i} \theta_{i}, y_{j} \theta_{j}\right\}=y_{i} y_{j} \theta_{i} \theta_{j} f_{i j}^{[q]}(\varrho(y))
$$

L'application

$$
\psi:\left(y_{1}, y_{2}, y_{3}\right) \longmapsto\left(y_{1} \theta_{1}(\varrho), y_{2} \theta_{2}(\varrho), y_{3} \theta_{3}(\varrho)\right)
$$

est un changement de coordonnées au voisinage de 0 puisque $\theta_{i}(0)=1$ pour tout $i$. En posant $z_{i}=y_{i} \theta_{i}(\varrho(y))$, on obtient :

$$
\left\{z_{i}, z_{j}\right\}=z_{i} z_{j} f_{i j}^{[q]}(\varrho(y))
$$

On doit exprimer $\varrho(y)$ en fonction des variables $z_{1}, z_{2}$ et $z_{3}$.

Mais auparavant, on va montrer qu'il existe une fonction $\bar{f}$ qui vaut 1 en zéro et un vecteur $\bar{V}$ non colinéaire à $(a, b, c)$ tels que

$$
\left(f_{23}^{[q]}(\varrho), f_{31}^{[q]}(\varrho), f_{12}^{[q]}(\varrho)\right)=\bar{f}(\varrho)(a, b, c)+\varrho^{q} \bar{V}
$$

Rappelons que $q$ est le plus petit entier tel que les fonctions $F_{i}$ n'aient pas leur jet d'ordre $q$ simultanément nul en zéro. Donc en faisant un 
développement limité des $F_{i}$ au voisinage de 0 , à l'ordre $(q-1)$ et en tenant compte du fait que $I_{0}=\lambda(a, b, c)$, il vient

$$
\left(f_{23}^{[q-1]}(\varrho), f_{31}^{[q-1]}(\varrho), f_{12}^{[q-1]}(\varrho)\right)=\left(\sum_{\ell=0}^{q-1} \frac{\bar{\lambda}_{\ell}}{\ell !} \varrho^{\ell}\right)(a, b, c) .
$$

Par ailleurs, d'après le lemme 2.2 la dérivée d'ordre $q$ en zéro de $F_{i}$ est égale à $q ! \mu_{i}$; donc on a :

$$
q !\left(\mu_{1}, \mu_{2}, \mu_{3}\right)=\left(F_{1}^{(q)}(0), F_{2}^{(q)}(0), F_{3}^{(q)}(0)\right) .
$$

Soit

$$
q !\left(\mu_{1}, \mu_{2}, \mu_{3}\right)=-\left(f_{23}^{(q)}(0), f_{31}^{(q)}(0), f_{12}^{(q)}(0)\right) \times\left(n_{1}, n_{2}, n_{3}\right),
$$

où le symbole $\times$ désigne le produit vectoriel usuel sur $\mathbb{R}^{3}$. Il s'en suit qu'il existe un réel $\kappa_{0}$ tel que :

$$
\frac{1}{q !}\left(f_{23}^{(q)}(0), f_{31}^{(q)}(0), f_{12}^{(q)}(0)\right)=\frac{1}{n_{3}}\left(\mu_{2},-\mu_{1}, 0\right)+\kappa_{0}\left(n_{1}, n_{2}, n_{3}\right) .
$$

Posons

$$
\bar{f}=\sum_{\ell=0}^{q-1} \frac{\bar{\lambda}_{\ell}}{\ell !} \varrho^{\ell}+\lambda \kappa_{0} \varrho^{q}=1+\lambda_{1} \varrho+\cdots+\lambda_{q} \varrho^{q} \quad \text { et } \quad \bar{V}=\frac{1}{n_{3}}\left(\mu_{2},-\mu_{1}, 0\right) .
$$

Il vient

$$
\left(f_{23}^{[q]}(\varrho), f_{31}^{[q]}(\varrho), f_{12}^{[q]}(\varrho)\right)=\bar{f}(\varrho)(a, b, c)+\varrho^{q} \bar{V}
$$

À présent, on va exprimer $\varrho(y)$ en fonction des $z_{i}$. En posant

$$
\theta=\theta_{1}^{n_{1}} \theta_{2}^{n_{2}} \theta_{3}^{n_{3}}
$$

on obtient

$$
\varrho(z)=\varrho(y) \theta(\varrho(y)) .
$$

Mais, l'application $t \mapsto t \theta(t)$ est un difféomorphisme local au voisinage de zéro car $\theta(0)=1$. Sa restriction à un intervalle centré en zéro de rayon $\epsilon$ (assez petit) admet une réciproque donnée par $t \mapsto t \beta(t)$, où $\beta$ est une fonction qui vaut 1 en zéro. Notons $\gamma$ cette réciproque, nous avons l'égalité

$$
\varrho(y)=\gamma(\varrho(z))
$$


Cette dernière égalité et la relation (12) donnent

$$
\left(f_{23}^{[q]}(\varrho(y)), f_{31}^{[q]}(\varrho(y)), f_{12}^{[q]}(\varrho(y))\right)=\bar{f} \circ \gamma(\varrho(z))(a, b, c)+\gamma^{q}(\varrho(z)) \bar{V} .
$$

On note

$$
f=\bar{f} \circ \gamma, \quad h=\gamma^{q}, \quad \bar{\Pi}^{2}=\frac{1}{n_{3}}\left(\mu_{1} z_{1} z_{3} \frac{\partial}{\partial z_{1}} \wedge \frac{\partial}{\partial z_{3}}+\mu_{2} z_{2} z_{3} \frac{\partial}{\partial z_{2}} \wedge \frac{\partial}{\partial z_{3}}\right) .
$$

Avec ces notations, les relations (11) et (13) impliquent :

$$
\psi_{*} \Pi=f(\varrho) \Pi^{2}+h(\varrho) \bar{\Pi}^{2} .
$$

La structure de Poisson diagonale $\bar{\Pi}^{2}$ n'est pas un multiple réel de $\Pi^{2}$ car s'il existait un réel $\delta$ tel que $\left(\mu_{2},-\mu_{1}, 0\right)=\delta(a, b, c)$, les $\mu_{i}$ seraient tous nuls puisque le coefficient $c$ est par hypothèse strictement positif. Ceci voudrait dire que les $F_{i}$ sont nulles, or ce cas est exclu. La fonction $h$ a un $(q-1)$-jet nul en zéro puisqu'on a

$$
h(\varrho)=\gamma^{q}(\varrho)=\varrho^{q} \beta^{q}(\varrho) \text { avec } \beta(0)=1 .
$$

D'où la proposition.

Nous venons de voir que si $\varrho$ est une fonction de Casimir alors $\Pi^{\prime}=f(\varrho) \Pi^{2}$. Sinon, il est formellement isomorphe à une structure de Poisson du type $\Pi^{\prime \prime \prime}=f(\varrho) \Pi^{2}+h(\varrho) \bar{\Pi}^{2}$.

Proposition 2.3. - Soit $\Pi^{\prime \prime}=f(\varrho) \Pi^{2}$ une structure de Poisson sur $\mathbb{R}^{m}, \Pi^{2}$ étant une structure de Poisson quadratique diagonalisable, $f$ une fonction non constante qui vaut 1 en zéro et $\varrho$ une fonction de Casimir pour $\Pi^{\prime \prime}$. Il existe un entier non nul $p$ et un changement de coordonnées $\Phi$ préservant 0 tels que:

$$
\Phi_{*} \Pi^{\prime \prime}=\left(1 \pm \varrho^{p}\right) \Pi^{2}
$$

Preuve. - Soient $\left(x_{1}, \ldots, x_{m}\right)$ des coordonnées nulles en 0 telles que

$$
\Pi^{\prime \prime}=f(\varrho(x)) \sum_{i<j} a_{i j} x_{i} x_{j} \frac{\partial}{\partial x_{i}} \wedge \frac{\partial}{\partial x_{j}} .
$$

Posons $y_{i}=x_{i} \theta_{i}(\varrho)$, avec $\theta_{i}(0)=1$ pour tout $i$. Il s'agit de montrer que l'on peut trouver des $\theta_{i}$ tels que le changement de coordonnées $\left(x_{1}, \ldots, x_{m}\right) \mapsto\left(y_{1}, \ldots, y_{m}\right)$ vérifie la proposition. Nous avons

$$
\begin{aligned}
& \quad\left\{y_{i}, y_{j}\right\}=\theta_{i} \theta_{j}\left\{x_{i}, x_{j}\right\}=a_{i j} \theta_{i} \theta_{j} x_{i} x_{j} f(\varrho(x))=a_{i j} y_{i} y_{j} f(\varrho(x)) . \\
& \text { TOME } 125-1997-\mathrm{N}^{\circ} 4
\end{aligned}
$$


Par un raisonnement identique à celui fait dans la preuve de la proposition précédente, on montre l'existence d'une fonction $\bar{\gamma}$ définie par $\bar{\gamma}: t \mapsto t \bar{\beta}(t)$, avec $\bar{\beta}(0)=1$ et telle que

$$
\varrho(x)=\bar{\gamma}(\varrho(y))
$$

Par suite,

$$
\left\{y_{i}, y_{j}\right\}=a_{i j} y_{i} y_{j} f \circ \bar{\gamma}(\varrho(y)) .
$$

Mais, la fonction $\bar{\gamma}$ dépend du choix $\operatorname{des} \theta_{i}$. On peut choisir les fonctions $\theta_{i}$ de façon qu'on ait :

$$
f \circ \bar{\gamma}(\varrho)=1 \pm \varrho^{p}
$$

En effet, d'une manière générale, on a le développement de Taylor

$$
f(t)=1+a_{p} t^{p}+a_{p+1} t^{p+1}+\cdots
$$

où $p$ est le plus petit entier tel que la dérivée d'ordre $p$ en zéro de $f$ ne soit pas nulle. Ceci peut encore s'écrire

$$
f(t)=1+\frac{a_{p}}{\left|a_{p}\right|}\left(t \sqrt[p]{\left|a_{p}\right|(1+t R(t))}\right)^{p} .
$$

Or, la fonction définie par

$$
\delta: t \longmapsto t \sqrt[p]{\left|a_{p}\right|(1+t R(t))}
$$

est un difféomorphisme local au voisinage de zéro. Donc, sa restriction à un intervalle centré en zéro et de rayon assez petit admet une fonction réciproque que l'on notera $\delta^{-1}$. En prenant $\bar{\gamma}=\delta^{-1}$, on a :

$$
f \circ \bar{\gamma}(\varrho)=1+\frac{a_{p}}{\left|a_{p}\right|} \varrho^{p}=1 \pm \varrho^{p}
$$

Ainsi, pour un choix convenable des fonctions $\theta_{i}$, on peut écrire :

$$
\left\{y_{i}, y_{j}\right\}=\left(1 \pm \varrho^{p}\right) a_{i j} y_{i} y_{j}
$$

D'où la proposition.

Revenons en dimension 3 et considérons la forme normale formelle donnée par :

$$
\Pi^{\prime \prime \prime}=f(\varrho) \Pi^{2}+h(\varrho) \bar{\Pi}^{2}
$$


avec $f(0)=1, \varrho(z)=z_{1}^{n_{1}} z_{2}^{n_{2}} z_{3}^{n_{3}}, h(\varrho)=\varrho^{q}+h_{q+1} \varrho^{q+1}+\cdots$ et

$$
\bar{\Pi}^{2}=\frac{1}{n_{3}}\left(\mu_{1} z_{1} z_{3} \frac{\partial}{\partial z_{1}} \wedge \frac{\partial}{\partial z_{3}}+\mu_{2} z_{2} z_{3} \frac{\partial}{\partial z_{2}} \wedge \frac{\partial}{\partial z_{3}}\right) .
$$

Proposition 2.4. - Il existe un changement de coordonnées $\psi$ fixant 0 et vérifiant

$$
\psi_{*} \Pi^{\prime \prime \prime}=\left(1+\alpha_{1} \varrho+\cdots+\alpha_{q-1} \varrho^{q-1}\right) \Pi^{2}+\varrho^{q} \widetilde{\Pi}^{2}
$$

où $\widetilde{\Pi}^{2}$ est une structure de Poisson diagonale qui n'est pas un multiple de $\Pi^{2}$.

Démonstration. - Nous cherchons un changement de coordonnées du type

$$
\psi:\left(z_{1}, z_{2}, z_{3}\right) \longmapsto\left(z_{1} \theta_{1}(\varrho), z_{2} \theta_{2}(\varrho), z_{3} \theta_{3}(\varrho)\right),
$$

avec $\theta_{i}(0)=1$. Pour les commodités du calcul nous noterons

$$
b_{12}=0, \quad b_{23}=\frac{\mu_{2}}{n_{3}}, \quad b_{31}=-\frac{\mu_{1}}{n_{3}} .
$$

Notons également $x_{i}=z_{i} \theta_{i}(\varrho)$. Alors

$$
\left\{x_{i}, x_{j}\right\}=z_{i} z_{j} \theta_{i} \theta_{j}\left(f(\varrho) a_{i j}+h(\varrho) b_{i j}+\varrho h(\varrho)\left(\sum_{\ell} b_{i \ell} n_{\ell} \frac{\theta_{j}^{\prime}}{\theta_{j}}-\sum_{\ell} b_{j \ell} n_{\ell} \frac{\theta_{i}^{\prime}}{\theta_{i}}\right)\right) .
$$

Ceci peut encore s'écrire

$$
\left\{x_{i}, x_{j}\right\}=x_{i} x_{j} \alpha_{i j}(\varrho(z))
$$

avec $\alpha_{i j}(\varrho)=f(\varrho) a_{i j}+h(\varrho) b_{i j}+\varrho h(\varrho)\left(\sum_{\ell} b_{i \ell} n_{\ell} \frac{\theta_{j}^{\prime}}{\theta_{j}}-\sum_{\ell} b_{j \ell} n_{\ell} \frac{\theta_{i}^{\prime}}{\theta_{i}}\right)$.

Afin d'obtenir une écriture synthétique des relations (14), nous adoptons la notation

$$
\vec{\alpha}(\varrho)=\left(\alpha_{23}(\varrho), \alpha_{31}(\varrho), \alpha_{12}(\varrho)\right) .
$$

En développant les composantes $\alpha_{i j}$ puis en remplaçant $\left(n_{1}, n_{2}, n_{3}\right)$ par $\lambda(a, b, c)$, nous obtenons

$$
\begin{aligned}
\vec{\alpha}(\varrho)=(f(\varrho) & \left.-\frac{\lambda}{n_{3}} \varrho h(\varrho)\left(\mu_{2} \frac{\theta_{1}^{\prime}}{\theta_{1}}-\mu_{1} \frac{\theta_{2}^{\prime}}{\theta_{2}}\right)\right)(a, b, c) \\
& +\frac{h(\varrho)}{n_{3}}\left(1+\varrho\left(n_{1} \frac{\theta_{1}^{\prime}}{\theta_{1}}+n_{2} \frac{\theta_{2}^{\prime}}{\theta_{2}}+n_{3} \frac{\theta_{3}^{\prime}}{\theta_{3}}\right)\right)\left(\mu_{2},-\mu_{1}, 0\right)
\end{aligned}
$$

TOME $125-1997-\mathrm{N}^{\circ} 4$ 
Posons

$$
\begin{aligned}
& C_{1}(\varrho)=f(\varrho)-\frac{\lambda}{n_{3}} \varrho h(\varrho)\left(\mu_{2} \frac{\theta_{1}^{\prime}}{\theta_{1}}-\mu_{1} \frac{\theta_{2}^{\prime}}{\theta_{2}}\right) \\
& C_{2}(\varrho)=h(\varrho)\left(1+\varrho\left(n_{1} \frac{\theta_{1}^{\prime}}{\theta_{1}}+n_{2} \frac{\theta_{2}^{\prime}}{\theta_{2}}+n_{3} \frac{\theta_{3}^{\prime}}{\theta_{3}}\right)\right) .
\end{aligned}
$$

Il vient alors

$$
\vec{\alpha}(\varrho)=C_{1}(\varrho)(a, b, c)+\frac{C_{2}(\varrho)}{n_{3}}\left(\mu_{2},-\mu_{1}, 0\right)
$$

Maintenant, il faut exprimer $C_{1}(\varrho(z))$ et $C_{2}(\varrho(z))$ en fonction de $\varrho(x)$; le raisonnement est identique à celui fait dans la preuve de la proposition 2.2 : si l'on pose $\eta=\theta_{1}^{n_{1}} \theta_{2}^{n_{2}} \theta_{3}^{n_{3}}$, alors

$$
\varrho(x)=\varrho(z) \eta(\varrho(z)) .
$$

Le fait que $\eta(0)=1$ implique l'existence d'une fonction $\xi$ définie au voisinage de zéro vérifiant $\xi(0)=1$ et telle que les deux fonctions $t \mapsto t \eta(t)$ et $t \mapsto t \xi(t)$ soient réciproques l'une de l'autre. Ainsi, nous avons

$$
\varrho(z)=\varrho(x) \xi(\varrho(x)) .
$$

Remplaçons $\varrho(z)$ par cette valeur dans les expressions de $C_{1}(\varrho(z))$ et $C_{2}(\varrho(z))$ et faisons un développement limité de ces fonctions en tenant compte du fait que $f(0)=1$ et $h$ est d'ordre $q$ en 0 ; il vient

$$
\begin{aligned}
& C_{1}(\varrho(z))=1+\alpha_{1} \varrho(x)+\cdots+\alpha_{q} \varrho^{q}(x)+\varrho^{q+1}(x) R_{1}(\varrho(x)) \\
& C_{2}(\varrho(z))=\left(\varrho(x) \sqrt[q]{1+\varrho(x) R_{2}(\varrho(x))}\right)^{q}
\end{aligned}
$$

Pour un bon choix des fonctions $\theta_{i}$, on a

$$
C_{1}(\varrho(z))=1+\alpha_{1} \varrho(x)+\cdots+\alpha_{q} \varrho^{q}(x), \quad C_{2}(\varrho(z))=\varrho^{q}(x)
$$

En effet, si l'on considère un changement de coordonnées $\psi$ qui est la composée de deux changements de coordonnées définis par

$$
\begin{aligned}
& \phi:\left(z_{1}, z_{2}, z_{3}\right) \longmapsto\left(z_{1} \gamma_{1}(\varrho), z_{2} \gamma_{2}(\varrho), z_{3} \gamma_{3}(\varrho)\right), \\
& \bar{\phi}:\left(z_{1}, z_{2}, z_{3}\right) \longmapsto\left(z_{1} \bar{\gamma}_{1}(\varrho), z_{2} \bar{\gamma}_{2}(\varrho), z_{3} \bar{\gamma}_{3}(\varrho)\right),
\end{aligned}
$$


avec la condition suivante : $\bar{\gamma}_{1} \bar{\gamma}_{2} \bar{\gamma}_{3}=1$. On montre, comme dans la preuve de la proposition 2.3, que l'on peut choisir les $\gamma_{i}$ de manière qu'on ait :

$$
C_{2} \circ \varrho=(\varrho \circ \phi)^{q}
$$

De même, pour un choix convenable des fonctions $\bar{\gamma}_{i}$, on a :

$$
C_{1} \circ \varrho=1+\alpha_{1}(\varrho \circ \psi)+\cdots+\alpha_{q}(\varrho \circ \psi)^{q}
$$

avec $\psi=\bar{\phi} \circ \phi$. Or, l'hypothèse $\bar{\gamma}_{1} \bar{\gamma}_{2} \bar{\gamma}_{3}=1$ entraîne la relation $\varrho \circ \bar{\phi}=\varrho$. Il vient :

$$
\varrho(x)=\varrho \circ \psi(z)=\varrho \circ \phi(z) .
$$

Par conséquent, les relations (16) et (15) donnent :

$$
C_{1}(\varrho(z))=1+\alpha_{1} \varrho(x)+\cdots+\alpha_{q} \varrho^{q}(x), \quad C_{2}(\varrho(z))=\varrho^{q}(x) .
$$

Ainsi, nous obtenons :

$$
\begin{aligned}
\vec{\alpha}(\varrho(z))=\left(1+\alpha_{1} \varrho(x)+\right. & \left.\cdots+\alpha_{q-1} \varrho^{q-1}(x)\right)(a, b, c) \\
& +\varrho^{q}(x)\left(\frac{1}{n_{3}}\left(\mu_{2},-\mu_{1}, 0\right)+\alpha_{q}(a, b, c)\right) .
\end{aligned}
$$

Notons

$$
\begin{aligned}
\widetilde{\Pi}^{2}=\alpha_{q} c x_{1} x_{2} \frac{\partial}{\partial x_{1}} \wedge \frac{\partial}{\partial x_{2}}+\left(\frac{\mu_{2}}{n_{3}}\right. & \left.+\alpha_{q} a\right) x_{2} x_{3} \frac{\partial}{\partial x_{2}} \wedge \frac{\partial}{\partial x_{3}} \\
& -\left(\frac{\mu_{1}}{n_{3}}-\alpha_{q} b\right) x_{3} x_{1} \frac{\partial}{\partial x_{3}} \wedge \frac{\partial}{\partial x_{1}} .
\end{aligned}
$$

Les relations (14) et la nouvelle expression de $\vec{\alpha}(\varrho(z))$ donnent

$$
\psi_{*} \Pi^{\prime \prime}=\left(1+\alpha_{1} \varrho+\cdots+\alpha_{q-1} \varrho^{q-1}\right) \Pi^{2}+\varrho^{q} \widetilde{\Pi}^{2} .
$$

Le tenseur $\widetilde{\Pi}^{2}$ n'est pas un multiple de $\Pi^{2}$ car $n_{3}^{-1}\left(\mu_{2},-\mu_{1}, 0\right)+\alpha_{q}(a, b, c)$ n'est pas colinéaire à $(a, b, c)$. Ce qui achève la démonstration.

Il découle des deux dernières propositions et du raisonnement qui précède l'énoncé du théorème 2.1 que toute structure de Poisson sur $\mathbb{R}^{3}$ du type $\Pi^{2}+P$ (où la perturbation $P$ a un 2 -jet nul en 0 ) est formellement isomorphe à $M_{0}, M_{1}, M_{2}$ ou $M_{3}$. Pour prouver complètement le théorème 2.1, il faut s'assurer que ces modèles ne sont pas isomorphes.

TOME $125-1997-\mathrm{N}^{\circ} 4$ 
Proposition 2.5. - Soit $\Pi=\left(1+\alpha_{1} \varrho+\cdots+\alpha_{q-1} \varrho^{q-1}\right) \Pi^{2}+\varrho^{q} \widetilde{\Pi}^{2}$ une structure de Poisson sur $\mathbb{R}^{3}$ avec $\widetilde{\Pi}^{2}$ qui n'est pas un multiple de $\Pi^{2}$. Alors $\Pi$ n'est isomorphe à aucune structure de Poisson du type $\Lambda=F \Pi^{2}$, où $F$ est une fonction de $\mathbb{R}^{3}$ telle que $F(0)=1$.

Preuve. - Raisonnons par l'absurde. Supposons qu'il existe un difféomorphisme $\varphi$ préservant l'origine tel que $\varphi_{*} \Pi=\Lambda$. Il envoie chaque feuille du feuilletage $\mathcal{F}_{\Pi}$ associé à $\Pi$ sur une feuille du feuilletage $\mathcal{F}_{\Lambda}$. L'aspect des ces feuilletages symplectiques montre que les composantes $\left(y_{1}, y_{2}, y_{3}\right)$ de $\varphi$ sont sous la forme

$$
y_{i}=x_{i} \theta_{i}\left(x_{1}, x_{2}, x_{3}\right) \quad \text { avec } \quad \theta_{i}(0) \neq 0 .
$$

On a :

$$
\left\{y_{i}, y_{j}\right\}=\theta_{i} \theta_{j}\left\{x_{i}, x_{j}\right\}+\theta_{i} x_{j}\left\{x_{i}, \theta_{j}\right\}+\theta_{j} x_{i}\left\{\theta_{i}, x_{j}\right\}+x_{i} x_{j}\left\{\theta_{i}, \theta_{j}\right\} .
$$

Notons $\tilde{a}_{i j}$ les coefficients de la structure de Poisson diagonale $\widetilde{\Pi}^{2}$. Notons également

$$
\begin{gathered}
H(\varrho)=1+\alpha_{1} \varrho+\cdots+\alpha_{q-1} \varrho^{q-1} \\
\widetilde{A}_{i} \cdot I=\sum_{j=1}^{n} \tilde{a}_{i j} I_{j}, \quad \Theta_{i}=\ln \left(\left|\theta_{i}\right|\right)=\sum_{I} \Theta_{i}^{I} x^{I} .
\end{gathered}
$$

Avec ces notations on peut écrire :

$$
\left\{y_{i}, y_{j}\right\}=y_{i} y_{j}\left(\frac{1}{x_{i} x_{j}}\left\{x_{i}, x_{j}\right\}+\frac{1}{x_{i}}\left\{x_{i}, \Theta_{j}\right\}+\frac{1}{x_{j}}\left\{\Theta_{i}, x_{j}\right\}+\left\{\Theta_{i}, \Theta_{j}\right\}\right) .
$$

Ce qui peut encore s'écrire

$$
\begin{aligned}
\left\{y_{i}, y_{j}\right\}=y_{i} y_{j}\left(H ( \varrho ) \left(a_{i j}+\sum_{I} x^{I}\left(A_{i} \cdot I \Theta_{j}^{I}\right.\right.\right. \\
\left.\left.-A_{j} \cdot I \Theta_{i}^{I}+\sum_{J+K=I} \Theta_{i}^{J} \Theta_{j}^{K} a_{r s} J_{r} K_{s}\right)\right) \\
+\varrho^{q}\left(\tilde{a}_{i j}+\sum_{I} x^{I}\left(\widetilde{A}_{i} \cdot I \Theta_{j}^{I}-\widetilde{A}_{j} \cdot I \Theta_{i}^{I}\right.\right. \\
\left.\left.\left.+\sum_{J+K=I} \Theta_{i}^{J} \Theta_{j}^{K} \tilde{a}_{r s} J_{r} K_{s}\right)\right)\right) .
\end{aligned}
$$

BULLETIN DE LA SOCIÉTÉ MATHÉMATIQUE DE FRANCE 
Par ailleurs, il résulte de l'hypothèse $\varphi_{*} \Pi=\Pi^{2}$ que le crochet $\left\{y_{i}, y_{j}\right\}$ vaut aussi $a_{i j} y_{i} y_{j} F$. Posons

$$
F=\sum F^{I} x^{I}
$$

et considérons uniquement les termes en $\varrho^{q}(x)$. En vertu du fait que $A_{i} . I$ est nul pour tout $I$ colinéaire à $I_{0}$, les termes en $\varrho^{q}(x)$ donnent :

$$
\sum_{\substack{0 \leq h \leq q-1 \\ 1 \leq r, s \leq 3}} \sum_{\substack{J+K=(q-h) I_{0} \\ 1}} \alpha_{h} \Theta_{i}^{J} \Theta_{j}^{K} a_{r s} J_{r} K_{s}+\tilde{a}_{i j}=a_{i j} F^{q I_{0}} .
$$

Mais, si $J+K$ est colinéaire à $I_{0}$, alors le terme $\sum_{r, s} a_{r s} J_{r} K_{s}$ est nul car c'est le produit mixte de trois vecteurs liés. En effet, on a :

$$
\left.\sum_{r, s} a_{r s} J_{r} K_{s}=\sum_{r}\left(A_{r} \cdot K\right) J_{r}=\operatorname{det}((a, b, c), J, K)\right) .
$$

Comme $I_{0}=\lambda(a, b, c)$, donc si $J+K$ est lié à $I_{0}$, on a nécessairement la nullité de $\sum_{r, s} a_{r s} J_{r} K_{s}$. Ainsi, le système (17) équivaut à

$$
\tilde{a}_{i j}=a_{i j} F^{q I_{0}}
$$

ce qui contredit l'hypothèse suivant laquelle $\widetilde{\Pi}^{2}$ n'est pas un multiple de $\Pi^{2}$. Par conséquent $\Pi$ et $\Lambda$ ne sont pas isomorphes.

À présent, nous allons montrer que l'entier $p$ qui intervient dans le modèle $M_{2}$ est un invariant.

Proposition 2.6. - Soient $\Pi=\left(1+\varepsilon \varrho^{p}\right) \Pi^{2}$ et $\Pi^{\prime}=\left(1+\varepsilon^{\prime} \varrho^{q}\right) \Pi^{2}$ deux structures de Poisson sur $\mathbb{R}^{3}$, avec $\varepsilon= \pm 1$ et $\varepsilon= \pm \varepsilon^{\prime}$. Si $\Pi$ et $\Pi^{\prime}$ sont isomorphes alors $p=q$.

Preuve. - Supposons que $p<q$ et qu'il existe un difféomorphisme $\varphi$ tel que $\varphi_{*} \Pi=\Pi^{\prime}$. Les composantes de $\varphi$ s'écrivent :

$$
\varphi_{i}=y_{i}=x_{i} \theta_{i}\left(x_{1}, x_{2}, x_{3}\right) \quad \text { avec } \quad \theta_{i}(0) \neq 0 \text {. }
$$

Un calcul analogue à celui fait dans la preuve de la proposition 2.5 montre qu'en posant

$$
\Theta_{i}=\ln \left|\theta_{i}\right|=\sum_{I} x^{I} \Theta_{i}^{I}
$$

TOME $125-1997-\mathrm{N}^{\circ} 4$ 
les crochets des composantes de $\varphi$ s'écrivent :

$$
\begin{aligned}
\left\{y_{i}, y_{j}\right\}=y_{i} y_{j}\left(1+\varepsilon \varrho^{p}\right)\left(a_{i j}+\sum_{I} x^{I}\left(A_{i} \cdot I \Theta_{j}^{I}-A_{j} \cdot I \Theta_{i}^{I}\right.\right. & \\
& \left.\left.+\sum_{\substack{J+K=I \\
r, s}} \Theta_{i}^{J} \Theta_{j}^{K} a_{r s} J_{r} K_{s}\right)\right) .
\end{aligned}
$$

De l'hypothèse $\varphi_{*} \Pi=\Pi^{\prime}$, il résulte que

$$
\left\{y_{i}, y_{j}\right\}=a_{i j} y_{i} y_{j}\left(1+\varepsilon^{\prime} \varrho^{q}(y)\right)=a_{i j} y_{i} y_{j}\left(1+\varepsilon^{\prime} \varrho^{q}(x)\left(\theta_{1}^{n_{1}} \theta_{2}^{n_{2}} \theta_{3}^{n_{3}}\right)^{q}\right) .
$$

Donc, si l'on considère seulement les termes en $\varrho^{p}(x)$, nous obtenons :

$$
\pm a_{i j}+\sum_{J+K=p I_{0}} \Theta_{i}^{J} \Theta_{j}^{K} a_{r s} J_{r} K_{s}=0
$$

Or, lorsque $J+K$ est colinéaire à $I_{0}$, on montre comme dans la preuve de la proposition 2.5 que $\sum_{r, s} a_{r s} J_{r} K_{s}$ est nul. Il s'en suit que $a_{i j}=0$, ce qui est absurde. Donc, nécessairement on a $p \geq q$. De la même manière on montre que $q \geq p$. D'où $p=q$.

REMARQUe. - En tenant un raisonnement similaire, on montre que $M_{2}$ n'est pas isomorphe à $M_{0}$. Ce même raisonnement permet de prouver la proposition qui suit.

Proposition 2.7. - Soient $\Pi=\left(1+\alpha_{m} \varrho^{m}+\cdots+\alpha_{p-1} \varrho^{p-1}\right) \Pi^{2}+\varrho^{p} \widetilde{\Pi}^{2}$ et $\Pi^{\prime}=\left(1+\beta_{m^{\prime}} \varrho^{m^{\prime}}+\cdots+\beta_{q-1} \varrho^{q-1}\right) \Pi^{2}+\varrho^{q} \bar{\Pi}^{2}$ deux structures de Poisson sur $\mathbb{R}^{3}$, où $\alpha_{m}$ et $\beta_{m^{\prime}}$ sont deux réels non nuls, $\widetilde{\Pi}^{2}$ et $\bar{\Pi}^{2}$ sont deux structures de Poisson quadratiques diagonales qui ne sont pas multiples de $\Pi^{2}$. Si $\Pi$ et $\Pi^{\prime}$ sont isomorphes on $a$ :

- $m=m^{\prime}$

- $p=q$,

- il existe deux réels $k_{1}$ et $k_{2}$ tels qu'on ait $\widetilde{\Pi}^{2}=k_{1} \Pi^{2}+k_{2} \bar{\Pi}^{2}$.

Remarque. - Les coefficients $a_{i j}$ d'une structure de Poisson quadratique diagonalisable sont des invariants (voir [Wa2]). Or, contrairement au modèle $M_{1}$, les coefficients $a, b$ et $c$ sont de même signe dans $M_{2}$ et $M_{3}$. Donc $M_{1}$ n'est isomorphe ni à $M_{2}$, ni à $M_{3}$. Il reste à montrer que $M_{1}$ n'est pas isomorphe à $M_{0}$. 
Pour tout $(\alpha, \beta) \in \mathbb{R}^{2}$, on note

$$
\begin{aligned}
\Pi_{\alpha \beta}=a y z \frac{\partial}{\partial y} \wedge \frac{\partial}{\partial z}+\left(b z x-\beta y^{m_{1}} z^{m_{2}+1}\right) \frac{\partial}{\partial z} \wedge \frac{\partial}{\partial x} \\
+\left(c x y+\alpha y^{m_{1}+1} z^{m_{2}}\right) \frac{\partial}{\partial x} \wedge \frac{\partial}{\partial y}
\end{aligned}
$$

avec $a<0 \leq b<c,\left(m_{1}, m_{2}\right)=-\frac{1}{a}(b, c) \in \mathbb{N} \times \mathbb{N}^{*}, m_{1}+m_{2} \geq 2$.

Proposition 2.8. - Si $\Pi_{\alpha \beta}$ et $\Pi_{\alpha^{\prime} \beta^{\prime}}$ sont isomorphes alors $(\alpha, \beta)=$ $k\left(\alpha^{\prime}, \beta^{\prime}\right)$, où $k$ est un réel non $n u l$.

Preuve. - Supposons qu'il existe un difféomorphisme $\varphi$ préservant l'origine et tel que $\varphi_{*} \Pi_{\alpha \beta}=\Pi_{\alpha^{\prime} \beta^{\prime}}$. Soit $\varphi^{(1)}$ la linéarisée de $\varphi$ en 0 . Comme $\Pi_{\alpha \beta}$ et $\Pi_{\alpha^{\prime} \beta^{\prime}}$ admettent $\Pi^{2}$ pour 2 -jet en 0 , on a $\varphi_{*}^{(1)} \Pi^{2}=\Pi^{2}$. $\mathrm{Vu}$ l'aspect des feuilles symplectiques de $\Pi^{2}$, on a

$$
\varphi^{(1)}(x, y, z)=\left(\delta_{1} x, \delta_{2} y, \delta_{3} z\right) \quad \text { avec } \quad \delta_{i} \in \mathbb{R}^{*}
$$

À présent, nous notons

$$
\psi=\left(\varphi^{(1)}\right)^{-1} \circ \varphi
$$

Le difféomorphisme $\psi$ transforme $\Pi_{\alpha \beta}$ en une structure de Poisson $\Pi_{\alpha^{\prime \prime} \beta^{\prime \prime}}$ : on a

$$
\Pi_{\alpha^{\prime \prime} \beta^{\prime \prime}}=\left(\varphi^{(1)}\right)_{*}^{-1} \Pi_{\alpha^{\prime} \beta^{\prime}}=\Pi^{2}+\frac{\delta_{2}^{m_{1}} \delta_{3}^{m_{2}}}{\delta_{1}} y^{\prime m_{1}} z^{\prime m_{2}} \frac{\partial}{\partial x^{\prime}}\left(\alpha^{\prime} y^{\prime} \frac{\partial}{\partial y^{\prime}}+\beta^{\prime} z^{\prime} \frac{\partial}{\partial z^{\prime}}\right)
$$

où l'on convient de poser :

$$
x^{\prime}=\frac{1}{\delta_{1}} x, \quad y^{\prime}=\frac{1}{\delta_{2}} y, \quad z^{\prime}=\frac{1}{\delta_{3}} z .
$$

Par identification, on a

$$
\left(\alpha^{\prime \prime}, \beta^{\prime \prime}\right)=\frac{\delta_{2}^{m_{1}} \delta_{3}^{m_{2}}}{\delta_{1}}\left(\alpha^{\prime}, \beta^{\prime}\right)
$$

Par la suite, on notera

$$
q=m_{1}+m_{2}+1
$$

TOME $125-1997-\mathrm{N}^{\circ} 4$ 
et on désignera par $\left(\psi^{-1}\right)^{[q-2]}$ le jet d'ordre $(q-2)$ en 0 de la réciproque de $\psi$. Posons :

$$
\eta=\left(\psi^{-1}\right)^{[q-2]} \circ \psi=I d+\eta^{(q-1)}+\cdots .
$$

On a :

$$
\eta_{*} \Pi_{\alpha \beta}=\Pi^{2}+\Pi_{\alpha^{\prime \prime} \beta^{\prime \prime}}^{(q)}+\cdots .
$$

Donc, en notant $\eta_{i}$, pour $i \in\{1,2,3\}$, les composantes de $\eta$, il vient :

$$
\left\{\begin{array}{l}
\left\{\eta_{1}, \eta_{2}\right\}_{\alpha \beta}=c \eta_{1} \eta_{2}+\alpha^{\prime \prime} \eta_{2}^{m_{1}+1} \eta_{3}^{m_{2}}+\cdots \\
\left\{\eta_{1}, \eta_{3}\right\}_{\alpha \beta}=-b \eta_{1} \eta_{2}+\beta^{\prime \prime} \eta_{2}^{m_{1}} \eta_{3}^{m_{2}+1}+\cdots
\end{array}\right.
$$

Posons

$$
\begin{aligned}
x & =x_{1}, \quad y=x_{2}, \quad z=x_{3}, \\
\eta_{i} & =x_{i}+\gamma_{i}, \quad \text { pour tout } i \in\{1,2,3\}, \\
\gamma_{i} & =\sum_{|I| \geq q-1} \gamma_{i}^{I} x^{I} .
\end{aligned}
$$

Considérons uniquement les termes en $y^{m_{1}+1} z^{m_{2}}$ (resp. en $y^{m_{1}} z^{m_{2}+1}$ ) dans la première équation (resp. dans la seconde équation) de $(\mathcal{S})$, il vient

$$
\begin{gathered}
\alpha y^{m_{1}+1} z^{m_{2}}+\gamma_{1}^{I_{q}}\left(-a m_{2}\right) y^{m_{1}+1} z^{m_{2}}=c \gamma_{1}^{I_{q}} y^{m_{1}+1} z^{m_{2}}+\alpha^{\prime \prime} y^{m_{1}+1} z^{m_{2}} \\
\beta y^{m_{1}} z^{m_{2}+1}+\gamma_{1}^{I_{q}}\left(a m_{1}\right) y^{m_{1}} z^{m_{2}+1}=-b \gamma_{1}^{I_{q}} y^{m_{1}} z^{m_{2}+1}+\beta^{\prime \prime} y^{m_{1}} z^{m_{2}+1}
\end{gathered}
$$

avec $I_{q}=\left(0, m_{1}, m_{2}\right)$. Compte tenu du fait que $(b, c)=-a\left(m_{1}, m_{2}\right)$, on trouve :

$$
(\alpha, \beta)=\left(\alpha^{\prime \prime}, \beta^{\prime \prime}\right)=\frac{\delta_{2}^{m_{1}} \delta_{3}^{m_{2}}}{\delta_{1}}\left(\alpha^{\prime}, \beta^{\prime}\right) .
$$

Ce qui achève la preuve.

Remarque. - Cette proposition montre que $M_{0}$ n'est pas isomorphe à $M_{1}$ et il résulte de sa preuve que si $\alpha$ et $\beta$ ne sont pas simultanément nuls alors $\Pi_{\alpha \beta}$ dépend uniquement du rapport de $\alpha$ par $\beta$. Nous pouvons donc supposer que $\beta=1$.

Le but de ce qui suit est de donner une version $C^{\infty}$ du théorème 2.1 .

BULLETIN DE LA SOCIÉTÉ MATHÉMATIQUE DE FRANCE 


\section{Action infinitésimale - Rotationnel d'un champ de $p$-vecteurs}

Dans la suite, les champs de vecteurs considérés s'annuleront tous à l'origine. Les structures de Poisson que l'on étudie s'avèrent être souvent du type $\Pi=X \wedge Y$, où $X$ et $Y$ sont deux champs de vecteurs commutants. On sera donc amené à utiliser le résultat suivant :

THÉORÈME 3.1 (voir $[\mathrm{Ch}]$ ). - Soit $\varphi$ (resp. $\left.\varphi_{0}\right)$ une action infinitésimale de $\mathbb{R}^{n}$ sur $\mathbb{R}^{m}$ donnée par les champs de vecteurs $X_{1}, \ldots, X_{n}$ (resp. $X_{1_{0}}, \ldots, X_{n_{0}}$ ) qui commutent deux à deux. On suppose que pour tout $i \in\{1, \ldots, n\}$ on $a$ :

$$
X_{i}=X_{i_{0}}+X_{i_{\infty}}
$$

où les $X_{i_{\infty}}$ sont des champs de vecteurs plats en 0 . On note $\varphi_{0}$ l'action infinitésimale donnée par $X_{1_{0}}, \ldots, X_{n_{0}}$. Si $\varphi_{0}$ est hyperbolique (faiblement ou fortement) alors il existe un $C^{\infty}$-difféomorphisme du type $\psi=\mathrm{id}_{\mathbb{R}^{m}}+\psi_{\infty}$ avec $\psi_{\infty}$ plat en 0 , qui transforme $\varphi$ en $\varphi_{0}$ c'est-à-dire que l'on $a$ :

$$
\psi_{*} X_{i}=X_{i_{0}}, \quad \forall i \in\{1, \ldots, n\} .
$$

À présent, nous allons introduire un champ de vecteurs attaché au tenseur de Poisson qui est appelé rotationnel ou champ de vecteurs modulaire.

Considérons une variété $M$ orientable; soit $\Omega$ une forme volume. L'application $\Omega^{b}$ de l'espace des champs de $p$-vecteurs dans celui des $(n-p)$ formes différentielles sur $M$ définie par $A \mapsto i_{A} \Omega$ est un isomorphisme d'espaces vectoriels.

Notons $\Omega^{\sharp}$ son isomorphisme réciproque et $D_{\Omega}=\Omega^{\sharp} \circ \mathrm{d} \circ \Omega^{\mathrm{b}}$ (où d est l'opérateur de différentiation extérieure).

DÉfinition. - On appelle rotationnel d'un champ de $p$-vecteurs $A$ relativement à $\Omega$, le champ de $(p-1)$-vecteurs $D_{\Omega}(A)$.

L'opérateur $D_{\Omega}$ est de carré nul, c'est-à-dire qu'il vérifie $D_{\Omega} \circ D_{\Omega}=0$. Le rotationnel d'un champ de vecteurs relativement à $\Omega$ est exactement la divergence de ce vecteurs par rapport à $\Omega$ et le rotationnel d'une structure de Poisson est un champ de vecteurs. En outre, le crochet de Lie de deux champs de vecteurs $X$ et $Y$ s'exprime par la relation

$$
[X, Y]=-D_{\Omega}(X \wedge Y)-D_{\Omega}(X) \wedge Y+X \wedge D_{\Omega}(Y) \text {. }
$$

Soient $\left(x_{1}, \ldots, x_{n}\right)$ des coordonnées locales définies dans un ouvert $U$. Posons :

$$
\Pi=\sum_{i<j} \Pi_{i j} \frac{\partial}{\partial x_{i}} \wedge \frac{\partial}{\partial x_{j}}, \quad \Omega_{0}=\mathrm{d} x_{1} \wedge \ldots \wedge \mathrm{d} x_{n}
$$

TOME $125-1997-\mathrm{N}^{\circ} 4$ 
Alors le rotationnel de $\Pi$ par rapport à $\Omega_{0}$ s'écrit localement

$$
D_{\Omega_{0}}(\Pi)=\sum_{i j} \frac{\partial \Pi_{i j}}{\partial x_{j}} \frac{\partial}{\partial x_{i}} .
$$

Si $\Pi$ est un tenseur de Poisson, alors ses rotationnels sont des isomorphismes infinitésimaux, c'est-à-dire qu'on a $\left[\Pi, D_{\Omega}(\Pi)\right]=0$ (le crochet $[$, étant celui de Schouten). Lorsque la variété est de dimension 3, l'identité de Jacobi $[\Pi, \Pi]=0$ équivaut à la relation $\Pi \wedge D_{\Omega}(\Pi)=0$.

Pour plus de détails sur cet opérateur, on pourra consulter par exemple [D-H].

Proposition 3.1. - Soient $\Pi$ et $\Pi_{0}$ deux structures de Poisson sur $\mathbb{R}^{m}$ ayant un 1-jet nul à l'origine et une partie quadratique diagonale, de rotationnels respectifs $X$ et $X_{0}$ relativement à la forme volume canonique $\Omega_{0}$ telles que

$$
\Pi=\Pi_{0}+\Pi_{\infty}
$$

où $\Pi_{\infty}$ est plat en 0 . On suppose qu'il existe deux champs de vecteurs $Y$, $Y_{0}$ hyperboliques en 0 , avec $Y-Y_{0}$ plat en 0 qui vérifient:

$$
\Pi=X \wedge Y, \quad \Pi_{0}=X_{0} \wedge Y_{0}
$$

Si $X_{0}$ et $Y_{0}$ donnent une action infinitésimale hyperbolique (fortement ou faiblement) de $\mathbb{R}^{2}$ alors il existe un $C^{\infty}$-difféomorphisme $\delta=\mathrm{id}_{\mathbb{R}^{m}}+\delta_{\infty}$, avec $\delta_{\infty}$ plat en 0 , tel que $\delta_{*} \Pi=\Pi_{0}$.

Pour démontrer cette proposition, on sert du théorème suivant :

ThÉORÈme 3.2 (voir $[\mathrm{R}]$ ). - Soient $X$ un champ de vecteurs sur $\mathbb{R}^{n}$ hyperbolique en 0 et $g$ une fonction plate en 0 . L'équation $X(f)=g$ est résoluble.

Démonstration de la proposition 3.1. - Soient $f$ et $g$ deux fonctions différentiables telles que $f(0)=1$ et $g(0)=0$. On pose

$$
\bar{X}=f X+g Y, \quad \bar{Y}=\frac{1}{f} Y .
$$

Il est clair que $\Pi=\bar{X} \wedge \bar{Y}$. Montrons que l'on peut choisir $f$ et $g$ de manière que le crochet des champs de vecteurs $\bar{X}$ et $\bar{Y}$ soit nul. Nous avons

$$
[\bar{X}, \bar{Y}]=[X, Y]-\frac{1}{f} Y(f) X-\frac{1}{f^{2}}(f X(f)+g Y(f)+f Y(g)) Y .
$$

BULLETIN DE LA SOCIÉTÉ MATHÉMATIQUE DE FRANCE 
Comme $D_{\Omega_{0}}(X)$ est nul, donc en utilisant la formule ci-dessus et en remplaçant $D_{\Omega_{0}}(Y)$ par $\operatorname{div}(Y)$, nous obtenons :

$$
[X, Y]=-D_{\Omega_{0}}(X \wedge Y)-D_{\Omega_{0}}(X) Y+D_{\Omega_{0}}(Y) X=(\operatorname{div}(Y)-1) X
$$

où $\Omega_{0}$ est la forme volume canonique sur $\mathbb{R}^{m}$.

Il vient donc

$$
[\bar{X}, \bar{Y}]=(\operatorname{div}(Y)-1-Y(\ln f)) X-\frac{1}{f^{2}}(f X(f)+Y(f g)) Y .
$$

Ainsi, pour que les champs de vecteurs $\bar{X}$ et $\bar{Y}$ commutent, il suffit que le système suivant soit satisfait :

$$
Y(\ln f)=\operatorname{div}(Y)-1, \quad Y(f g)=-f X(f) .
$$

Si $\operatorname{div}(Y)-1$ est plate en 0 , ce système est résoluble. En effet, supposons pour l'instant que $\operatorname{div}(Y)-1$ est plate en 0 . Alors le théorème 3.2 garantit l'existence d'une fonction $f=1+f_{\infty}$, avec $f_{\infty}$ plate en 0 vérifiant la première équation de $(S)$. Ce même théorème permet d'affirmer que pour une telle fonction $f$, il existe une solution $g$ de la seconde équation de $(S)$ plate en 0 . Par conséquent, il suffit de montrer que $\operatorname{div}(Y)-1$ est plate en 0. Montrons-le par l'absurde. On a

$$
[X, Y]=(\operatorname{div}(Y)-1) X
$$

D'autre part, en utilisant les décompositions $X=X_{0}+X_{\infty}, Y=$ $Y_{0}+Y_{\infty}$, avec $X_{\infty}$ et $Y_{\infty}$ plats en 0 , on voit que l'on peut écrire le crochet de $X$ et $Y$ sous la forme

$$
[X, Y]=\left[X_{0}, Y_{0}\right]+[X, Y]_{\infty}
$$

où $[X, Y]_{\infty}$ est un champ de vecteurs plat en 0 . Compte tenu du fait que $X_{0}$ et $Y_{0}$ commutent, il vient :

$$
[X, Y]=[X, Y]_{\infty}
$$

Des relations (18) et (19) on tire

$$
(\operatorname{div}(Y)-1) X=[X, Y]_{\infty} .
$$

Il résulte de $(20)$ que $\operatorname{div}(Y)-1$ est plat en 0 . Par suite, les champs de vecteurs $\bar{X}$ et $\bar{Y}$ commutent. En outre, par construction, la linéarisée de

TOME $125-1997-\mathrm{N}^{\circ} 4$ 
l'action infinitésimale $\bar{\phi}$ donnée par $\bar{X}$ et $\bar{Y}$ coïncide avec celle de l'action infinitésimale hyperbolique donnée par $X_{0}$ et $Y_{0}$; par conséquent $\bar{\phi}$ est hyperbolique. Le théorème de Chaperon s'applique : il montre l'existence d'un $C^{\infty}$-difféomorphisme

$$
\delta=\operatorname{id}_{\mathbb{R}^{m}}+\delta_{\infty}
$$

avec $\delta_{\infty}$ plat en 0 , tel que $\delta_{*} \bar{X}=X_{0}$ et $\delta_{*} \bar{Y}=Y_{0}$. D'où

$$
\delta_{*} \Pi=\delta_{*} \bar{X} \wedge \delta_{*} \bar{Y}=\Pi_{0},
$$

ce qui achève la démonstration.

\section{Modèles locaux en classe $C^{\infty}$}

THÉORÈme 4.1. - Soit $\Pi$ une structure de Poisson sur $\mathbb{R}^{3}$ dont le jet d'ordre 1 est nul à l'origine. On suppose qu'il existe des coordonnées $\left(x_{1}, x_{2}, x_{3}\right)$ nulles en 0 telles le 2-jet de $\Pi$ en 0 s'écrit

$$
\Pi^{2}=c x_{1} x_{2} \frac{\partial}{\partial x_{1}} \wedge \frac{\partial}{\partial x_{2}}+a x_{2} x_{3} \frac{\partial}{\partial x_{2}} \wedge \frac{\partial}{\partial x_{3}}+b x_{3} x_{1} \frac{\partial}{\partial x_{3}} \wedge \frac{\partial}{\partial x_{1}},
$$

les coefficients $a, b$ et $c$ étant deux à deux distincts. On suppose également que si l'un des réels $a, b$ ou $c$ est nul, les deux autres sont de signe contraire. Alors $\Pi$ est isomorphe en classe $C^{\infty}$ à l'un des quatre modèles locaux du théorème 2.1 .

Pour établir la version $C^{\infty}$ du théorème 2.1, il suffit de montrer que les modèles locaux obtenus satisfont les hypothèses de la proposition 3.1. Considérons une structure de Poisson $\Pi=\Pi_{0}+\Pi_{\infty}$, où $\Pi_{0}$ désigne l'un des quatre modèles locaux polynomiaux et $\Pi_{\infty}$ un champ de bi-vecteurs plat à l'origine. Dans la suite, nous noterons

$$
\lambda_{1}=c-b, \quad \lambda_{2}=a-c, \quad \lambda_{3}=b-a .
$$

Nous désignons par $X$ (resp. $X_{0}$ ) le rotationnel de $\Pi$ (resp. $\Pi_{0}$ ) relativement à la forme volume canonique $\Omega_{0}$ de $\mathbb{R}^{3}$. Comme par hypothèse aucun des $\lambda_{i}$ n'est nul, donc tout champ de vecteurs dont la linéarisée est donnée par

$$
X^{(1)}=\lambda_{1} x \frac{\partial}{\partial x}+\lambda_{2} y \frac{\partial}{\partial y}+\lambda_{3} z \frac{\partial}{\partial z}
$$

est hyperbolique. Compte tenu du fait que l'identité de Jacobi pour les structures de Poisson $\Pi$ et $\Pi_{0}$ s'écrit respectivement $\Pi \wedge X=0$ et $\Pi_{0} \wedge X_{0}=0$, il existe des champs de vecteurs $Y$ et $Y_{0}$ tels que

$$
\Pi=X \wedge Y, \quad \Pi_{0}=X_{0} \wedge Y_{0} .
$$

Pour appliquer la proposition 3.1, nous voyons d'abord s'il est possible de choisir un champ de vecteurs $Y_{0}$ hyperbolique en 0 , tel que $X_{0}$ et $Y_{0}$ donnent une action infinitésimale hyperbolique (fortement ou faiblement). 
Nous emploierons le lemme qui suit :

Lemme 4.1. - Soit $\Pi$ une structure de Poisson sur $\mathbb{R}^{m}$ de rotationnel $X$ relativement à $\Omega_{0}$. On suppose que la linéarisée de $X$ en 0 est un difféomorphisme local au voisinage de 0 . Alors tout champ de vecteurs à divergence constante (relativement à $\Omega_{0}$ ) qui vérifie la relation $\Pi=X \wedge Y$, commute avec $X$.

Preuve. - On a :

$$
[X, Y]=(\operatorname{div}(Y)-1) X
$$

Posons $\operatorname{div}(Y)-1=\mu$ et considérons uniquement les termes linéaires, nous obtenons

$$
\left[X^{(1)}, Y^{(1)}\right]=\mu X^{(1)}
$$

où $X^{(1)}$ et $Y^{(1)}$ sont respectivement les linéarisées en 0 de $X$ et $Y$. Par hypothèse, $X^{(1)}$ est un difféomorphisme local au voisinage de 0 ; donc sa restriction à une boule centrée en 0 de rayon assez petit admet une réciproque que l'on note $Z^{(1)}$. On a alors :

$$
Z^{(1)}\left(X^{(1)} Y^{(1)}-Y^{(1)} X^{(1)}\right)=\mu \mathrm{id}
$$

ou encore

$$
Y^{(1)}-Z^{(1)} Y^{(1)} X^{(1)}=\mu \text { id } .
$$

Par passage aux traces, on obtient la nullité de $\mu$. D'où le lemme.

Maintenant, nous allons examiner successivement les quatre cas.

A) On suppose que $\Pi_{0}=\Pi^{2}$. - Le rotationnel de $\Pi_{0}$ relativement à $\Omega_{0}$ est :

$$
X^{(1)}=\lambda_{1} x \frac{\partial}{\partial x}+\lambda_{2} y \frac{\partial}{\partial y}+\lambda_{3} z \frac{\partial}{\partial z} .
$$

Soit $k$ un réel non nul, posons

$$
\begin{gathered}
\left(K_{1}, K_{2}, K_{3}\right)=\left(k \lambda_{1}, \frac{c}{\lambda_{1}}+k \lambda_{2}, \frac{-b}{\lambda_{1}}+k \lambda_{3}\right), \\
Y_{0}=K_{1} x \frac{\partial}{\partial x}+K_{2} y \frac{\partial}{\partial y}+K_{3} z \frac{\partial}{\partial z}
\end{gathered}
$$

On a la relation $\Pi^{2}=X^{(1)} \wedge Y_{0}$. On choisit un réel $k$ tel qu'aucun des $K_{i}$ ne soit nul; alors $Y_{0}$ est hyperbolique en 0 . D'autre part, $X^{(1)}$

$$
\text { TOME } 125-1997-\mathrm{N}^{\circ} 4
$$


et $Y_{0}$ commutent; ils donnent une action infinitésimale $\varphi_{0}$ dont les poids sont les applications définies sur $\mathbb{R}^{2}$ par

$$
\begin{aligned}
& P_{1}\left(v_{1}, v_{2}\right)=\exp \left(\lambda_{1} v_{1}+K_{1} v_{2}\right), \\
& P_{2}\left(v_{1}, v_{2}\right)=\exp \left(\lambda_{2} v_{1}+K_{2} v_{2}\right), \\
& P_{3}\left(v_{1}, v_{2}\right)=\exp \left(\lambda_{3} v_{1}+K_{3} v_{2}\right) .
\end{aligned}
$$

Dans la suite, on identifiera les $P_{i}$ à des covecteurs en posant :

$$
P_{1}=\left(\lambda_{1}, K_{1}\right), \quad P_{2}=\left(\lambda_{2}, K_{2}\right), \quad P_{3}=\left(\lambda_{3}, K_{3}\right) .
$$

Si $a, b$ et $c$ sont non nuls, ces poids sont deux à deux indépendants puisqu'on a :

$$
\operatorname{det}\left(P_{2}, P_{3}\right)=a \neq 0, \quad \operatorname{det}\left(P_{3}, P_{1}\right)=b \neq 0, \quad \operatorname{det}\left(P_{1}, P_{2}\right)=c \neq 0 .
$$

Donc, dans ce cas $\varphi_{0}$ est une action infinitésimale fortement hyperbolique.

Lorsque l'un des réels $a, b$ ou $c$ est nul, $\varphi_{0}$ est une action infinitésimale faiblement hyperbolique. En effet, supposons par exemple que $b=0$. Les valeurs propres de $X^{(1)}$ s'écrivent $\lambda_{1}=c, \lambda_{2}=a-c$ et $\lambda_{3}=-a$. Les poids de $\varphi_{0}$ sont :

$$
P_{1}=(c, k c), \quad P_{2}=\left(\lambda_{2}, 1+k \lambda_{2}\right), \quad P_{3}=(-a,-k a) .
$$

Nous avons l'égalité $a P_{1}=-c P_{3}$. Mais, par hypothèse $a$ et $c$ sont de signe contraire, donc le segment $\left[P_{1}, P_{3}\right]$ ne contient pas l'origine. Par ailleurs, on a

$$
\operatorname{det}\left(P_{2}, P_{3}\right)=a \neq 0, \quad \operatorname{det}\left(P_{1}, P_{2}\right)=c \neq 0 .
$$

Par conséquent, $\varphi_{0}$ est faiblement hyperbolique. La proposition 3.1 s'applique : il existe un changement de coordonnées $\varphi$ de classe $C^{\infty}$ au voisinage de l'origine tel que $\varphi_{*} \Pi=\Pi^{2}$.

B) On suppose $\alpha \in \mathbb{R}, a<0$ et $\left(m_{1}, m_{2}\right)=-\frac{1}{a}(b, c) \in \mathbb{N}^{2}$ et

$$
\Pi_{0}=\Pi^{2}+y^{m_{1}} z^{m_{2}} \partial x \wedge(\alpha y \partial y+z \partial z) .
$$

Le rotationnel de $\Pi_{0}$ relativement à $\Omega_{0}$ est :

$$
X_{0}=X^{(1)}+\left(\alpha\left(m_{1}+1\right)+\left(m_{2}+1\right)\right) y^{m_{1}} z^{m_{2}} \frac{\partial}{\partial x} .
$$

Soit :

$$
X_{0}=X^{(1)}-\frac{1}{a}\left(\alpha \lambda_{3}-\lambda_{2}\right) y^{m_{1}} z^{m_{2}} \frac{\partial}{\partial x} .
$$

Il faut envisager les deux cas $\alpha \lambda_{3}-\lambda_{2} \neq 0$ et $\alpha \lambda_{3}-\lambda_{2}=0$. 
$\left.B_{1}\right)$ Cas où $\alpha \lambda_{3}-\lambda_{2} \neq 0$. - Posons

$$
\begin{aligned}
K_{2} & =-\frac{\alpha a}{\alpha \lambda_{3}-\lambda_{2}}, \quad K_{3}=-\frac{a}{\alpha \lambda_{3}-\lambda_{2}} \\
K_{1} & =\frac{\lambda_{1} K_{3}+b}{\lambda_{3}}=\frac{\lambda_{1} K_{2}-c}{\lambda_{2}} \\
Y_{0} & =K_{1} x \frac{\partial}{\partial x}+K_{2} y \frac{\partial}{\partial y}+K_{3} z \frac{\partial}{\partial z}
\end{aligned}
$$

On la relation $\Pi_{0}=X_{0} \wedge Y_{0}$, de plus le lemme précédent montre que $X_{0}$ et $Y_{0}$ commutent. Ils donnent une action infinitésimale $\varphi_{0}$ ayant pour poids :

$$
P_{1}=\left(\lambda_{1}, K_{1}\right), \quad P_{2}=\left(\lambda_{2}, K_{2}\right), \quad P_{3}=\left(\lambda_{3}, K_{3}\right) .
$$

Si $a, b$ et $c$ sont non nuls, les poids sont deux à deux indépendants. Donc $\varphi_{0}$ est fortement hyperbolique. En revanche, si l'un des réels $a, b$ ou $c$ est nul, $\varphi_{0}$ est faiblement hyperbolique. Supposons que $b=0$; les poids de $\varphi$ deviennent :

$$
P_{1}=\left(c,-\frac{c}{\alpha a+\lambda_{2}}\right), \quad P_{2}=\left(\lambda_{2}, \frac{\alpha a}{\alpha a+\lambda_{2}}\right), \quad P_{3}=\left(-a, \frac{a}{\alpha a+\lambda_{2}}\right) .
$$

On voit que $a P_{1}=-c P_{3}$, mais le segment $\left[P_{1}, P_{3}\right]$ ne contient pas l'origine puisque $a$ et $c$ ne sont pas de même signe. Et comme on a

$$
\operatorname{det}\left(P_{1}, P_{2}\right)=c \neq 0, \quad \operatorname{det}\left(P_{2}, P_{3}\right)=a \neq 0,
$$

l'action infinitésimale $\varphi$ est faiblement hyperbolique.

$B_{2}$ ) Cas où $\alpha \lambda_{3}-\lambda_{2}=0$. - Pour tout $k$ fixé, le champ de vecteurs $Y_{0}$ donné par

$$
Y_{0}=-\frac{1}{\lambda_{3}} y^{m_{1}} z^{m_{1}} \frac{\partial}{\partial x}+\frac{c}{\lambda_{1}} y \frac{\partial}{\partial y}-\frac{b}{\lambda_{1}} z \frac{\partial}{\partial z}+k X^{(1)}
$$

vérifie $\Pi_{0}=X_{0} \wedge Y_{0}$. D'après le lemme 4.1 ce champ de vecteurs commute avec $X_{0}$. On choisira un réel $k$ tel que $k\left(k \lambda_{2} \lambda_{1}+c\right)\left(k \lambda_{3} \lambda_{1}-b\right) \neq 0$. Ainsi, on montre comme dans la partie $\mathrm{A}$ ), que l'action infinitésimale donnée par $X_{0}$ et $Y_{0}$ est hyperbolique. Les hypothèses de la proposition 3.1 sont satisfaites. On en déduit que toute structure de Poisson $\Pi=\Pi_{0}+\Pi_{\infty}$, avec $\Pi_{\infty}$ plat en 0 , est $C^{\infty}$-isomorphe à $\Pi_{0}$.

C) On suppose $\Pi_{0}=\left(1+\varepsilon \varrho^{p}\right) \Pi^{2}$, avec $\varepsilon= \pm 1, \varrho(x, y, z)=x^{n_{1}} y^{n_{2}} z^{n_{3}}$ et $I_{0}=\lambda(a, b, c) \in \mathbb{N}^{* 3}$. - Le rotationnel de $\Pi_{0}$ relativement à $\Omega_{0}$ est :

$$
X_{0}=\left(1+\varepsilon \varrho^{p}\right) X^{(1)} \text {. }
$$

TOME $125-1997-\mathrm{N}^{\circ} 4$ 
En tenant un raisonnement identique à celui fait dans $\mathrm{A}$ ), on voit que la proposition 3.1 s'applique. Par conséquent toute structure de Poisson $\Pi=\Pi_{0}+\Pi_{\infty}$, avec $\Pi_{\infty}$ plat à l'origine, est $C^{\infty}$-isomorphe à $\Pi_{0}$.

D) On suppose que $\Pi_{0}=\left(1+\alpha_{1} \varrho+\cdots+\alpha_{q-1} \varrho^{q-1}\right) \Pi^{2}+\varrho^{q} \widetilde{\Pi}^{2}\left(\widetilde{\Pi}^{2}\right.$ étant une structure de Poisson quadratique diagonale qui n'est pas un multiple $\operatorname{de} \Pi^{2}$ et $\left.q \in \mathbb{N}\right)$. - On a

$$
X_{0}=\left(1+\alpha_{1} \varrho+\cdots+\alpha_{q-1} \varrho^{q-1}\right) X^{(1)}+\varrho^{q} \widetilde{X}^{(1)}+q \varrho^{q} \widetilde{Y},
$$

où l'on convient de noter :

$$
\begin{gathered}
\widetilde{\Pi}^{2}=\tilde{c} x y \frac{\partial}{\partial x} \wedge \frac{\partial}{\partial y}+\tilde{a} y z \frac{\partial}{\partial y} \wedge \frac{\partial}{\partial z}+\tilde{b} z x \frac{\partial}{\partial z} \wedge \frac{\partial}{\partial y}, \\
\tilde{X}^{(1)}=(\tilde{c}-\tilde{b}) x \frac{\partial}{\partial x}+(\tilde{a}-\tilde{c}) y \frac{\partial}{\partial y}+(\tilde{b}-\tilde{a}) z \frac{\partial}{\partial z} \\
\tilde{Y}=\left(\tilde{c} n_{2}-\tilde{b} n_{3}\right) x \frac{\partial}{\partial x}+\left(\tilde{a} n_{3}-\tilde{c} n_{1}\right) y \frac{\partial}{\partial y}+\left(\tilde{b} n_{1}-\tilde{a} n_{2}\right) z \frac{\partial}{\partial z} .
\end{gathered}
$$

Introduisons les notations suivantes :

$$
\mathcal{V}=(\tilde{a}, \tilde{b}, \tilde{c}), \quad \mathcal{I}=(1,1,1), \quad I_{0}=\left(n_{1}, n_{2}, n_{3}\right)=\lambda(a, b, c) .
$$

Supposons que les vecteurs $\mathcal{I}, I_{0}$ et $\mathcal{V}$ sont linéairement indépendants et posons :

$$
Y_{0}=\frac{1}{\operatorname{det}\left(\mathcal{I}, I_{0}, \mathcal{V}\right)} \tilde{Y}
$$

Alors on a la relation $\Pi_{0}=X_{0} \wedge Y_{0}$. Le lemme 4.1 assure la commutativité des champs de vecteurs $X_{0}$ et $Y_{0}$. Ces vecteurs donnent une action infinitésimale $\varphi_{0}$ hyperbolique. Nous appliquons la proposition 3.1 qui dit que $\Pi$ est isomorphe à $\Pi_{0}$.

Cependant, si ces vecteurs sont liés, on ne peut plus appliquer cette proposition. En effet, $\operatorname{si} \operatorname{det}\left(\mathcal{I}, I_{0}, \mathcal{V}\right)=0$ et si l'un des coefficients $\alpha_{i}$ est non nul, alors aucun des champs de vecteurs $Y_{0}$ vérifant $\Pi_{0}=X_{0} \wedge Y_{0}$ ne commute avec $X_{0}$. Pour franchir cette difficulté, nous allons suivre une autre méthode. Nous utilisons le lemme 4.2 qui suit.

Dans la suite, nous nous placerons toujours dans le cas où la structure de Poisson $\Pi$ s'écrit :

$$
\Pi=\left(1+\alpha_{1} \varrho+\cdots+\alpha_{q-1} \varrho^{q-1}\right) \Pi^{2}+\varrho^{q} \widetilde{\Pi}^{2}+\Pi_{\infty}=\Pi_{0}+\Pi_{\infty} .
$$

Nous avons le lemme suivant : 
Lemme 4.2. - Il existe un changement de coordonnées $\xi$ de classe $C^{\infty}$ au voisinage de l'origine, du type $\xi=\mathrm{id}_{\mathbb{R}^{3}}+\xi_{\infty}$, avec $\xi_{\infty}$ plat en 0 et tel que $\xi_{*} \Pi$ soit une structure de Poisson diagonale.

Preuve. - Soient $r_{2}$ et $r_{3}$ deux réels positifs. On considère le champ de vecteurs linéaire diagonal donné par

$$
Y^{(1)}=-\left(b r_{2}+c r_{3}\right) x \frac{\partial}{\partial x}+a r_{2} y \frac{\partial}{\partial y}+a r_{3} z \frac{\partial}{\partial z} \text {. }
$$

La trace de $Y^{(1)}$ est négative; en outre le champ de vecteurs $\bar{Y}^{(1)}=$ $Y^{(1)} / \operatorname{tr}\left(Y^{(1)}\right)$ vérifie la relation :

$$
\Pi^{2}=X^{(1)} \wedge \bar{Y}^{(1)}
$$

Soit $Y$ un champ de vecteurs satisfaisant la relation $\Pi=X \wedge Y$ (X étant le rotationnel de $\Pi$ ). Nous pouvons supposer qu'il existe un champ de vecteurs $Y_{0}$ polynomial en $\varrho$ tel que $Y_{\infty}=Y-Y_{0}$ soit plat en 0 .

Quitte à modifier $Y$ en lui ajoutant un terme $k X$ (avec $k \in \mathbb{R}$ ) on peut toujours supposer que la linéarisée de $Y$ en 0 est égale à $\bar{Y}^{(1)}$. Notons que $\bar{Y}^{(1)}$ possède une valeur propre positive notée $\mu_{1}$ et deux valeurs propres négatives que l'on note $\mu_{2}$ et $\mu_{3}$.

On peut donc appliquer un résultat de Sternberg qui garantit l'existence d'un changement de coordonnées $\eta=\mathrm{id}+\eta_{\infty}$, avec $\eta_{\infty}$ plat en 0 , tel que $\eta_{*} Y=Y_{0}$. On en déduit

$$
\eta_{*} \Pi=\eta_{*} X \wedge \eta_{*} Y=\eta_{*}\left(D_{\Omega_{0}}(\Pi)\right) \wedge \eta_{*} Y
$$

On sait que, si l'on note $J \eta^{-1}$ le Jacobien de la réciproque de $\eta$, on a la relation

$$
J \eta^{-1} \eta_{*}\left(D_{\Omega_{0}}(\Pi)\right)=D_{\Omega_{0}}\left(J \eta^{-1} \eta_{*} \Pi\right)
$$

On tire

$$
J \eta^{-1} \eta_{*} \Pi=D_{\Omega_{0}}\left(J \eta^{-1} \eta_{*} \Pi\right) \wedge \eta_{*} Y
$$

Posons

$$
\Pi^{\prime}=J \eta^{-1} \eta_{*} \Pi, \quad X^{\prime}=D_{\Omega_{0}}\left(\Pi^{\prime}\right), \quad Y^{\prime}=\eta_{*} Y .
$$

Le champ de bivecteurs $\Pi^{\prime}$ est un tenseur de Poisson car c'est le produit d'un tenseur de Poisson sur $\mathbb{R}^{3}$ par une fonction. En outre, on a les relations :

$$
\Pi^{\prime}=X^{\prime} \wedge Y^{\prime}, \quad \eta_{*} \Pi=\frac{1}{J \eta^{-1}} \Pi^{\prime} .
$$

TOME $125-1997-\mathrm{N}^{\circ} 4$ 
Par conséquent, pour montrer que l'hypersurface $\{x=0\}$ est invariante pour $\eta_{*} \Pi$ (c'est-à-dire qu'elle est tangente au feuilletage associé à $\eta_{*} \Pi$ ), il suffit de prouver qu'elle est invariante pour $\Pi^{\prime}$. On a :

$$
\Pi^{\prime}=f(\varrho) \Pi^{2}+\varrho^{q} \widetilde{\Pi}^{2}+\Pi_{\infty}
$$

avec $\Pi_{\infty}^{\prime}$ plat en 0 . Désignons par $\Pi_{1}^{\prime}, X_{1}^{\prime}$ et $Y_{1}^{\prime}$ respectivement les restrictions à l'hypersurface $\{x=0\}$ de $\Pi^{\prime}, X^{\prime}$ et $Y^{\prime}$. Nous avons

$$
\begin{aligned}
\Pi_{1}^{\prime} & =a y z \frac{\partial}{\partial y} \wedge \frac{\partial}{\partial z}+\Pi_{1_{\infty}}^{\prime}, \\
X_{1}^{\prime} & =\lambda_{2} y \frac{\partial}{\partial y}+\lambda_{3} z \frac{\partial}{\partial z}+X_{1_{\infty}}^{\prime}, \\
Y_{1}^{\prime} & =\mu_{2} y \frac{\partial}{\partial y}+\mu_{3} z \frac{\partial}{\partial z}
\end{aligned}
$$

La relation $\Pi^{\prime}=X^{\prime} \wedge Y^{\prime}$ implique

$$
\Pi_{1}^{\prime}=X_{1}^{\prime} \wedge Y_{1}^{\prime}, \quad \Pi_{1}^{\prime} \wedge Y_{1}^{\prime}=0 .
$$

Notons $P_{i j}(y, z)$ les composantes de $\Pi_{1_{\infty}}^{\prime}$. Le système $(\Sigma)$ donnent les équations

$$
\begin{aligned}
\mu_{2} y P_{31}+\mu_{3} z P_{12} & =0, \\
\mu_{2} y\left(\frac{\partial P_{12}}{\partial y}+\frac{\partial P_{13}}{\partial z}\right) & =P_{12}, \\
\mu_{3} z\left(\frac{\partial P_{12}}{\partial y}+\frac{\partial P_{13}}{\partial z}\right) & =P_{13} .
\end{aligned}
$$

Dérivons (22) par rapport à $z$; on tire :

$$
\mu_{2} y \frac{\partial P_{13}}{\partial z}=\mu_{3} P_{12}+\mu_{3} z \frac{\partial P_{12}}{\partial z} .
$$

En portant ceci dans (23), on obtient :

$$
\mu_{2} y \frac{\partial P_{12}}{\partial y}+\mu_{3} z \frac{\partial P_{12}}{\partial z}=\left(1-\mu_{3}\right) P_{12} .
$$

De même, les relations (22) et (24) donnent

$$
\mu_{2} y \frac{\partial P_{13}}{\partial y}+\mu_{3} z \frac{\partial P_{13}}{\partial z}=\left(1-\mu_{2}\right) P_{12}
$$

BULLETIN DE LA SOCIÉTÉ MATHÉMATIQUE DE FRANCE 
ou encore

$$
Y_{1}^{\prime}\left(P_{12}\right)=\left(1-\mu_{3}\right) P_{12}, \quad Y_{1}^{\prime}\left(P_{13}\right)=\left(1-\mu_{2}\right) P_{13}
$$

On en déduit que $P_{12}$ et $P_{13}$ sont nulles. Ce qui montre que le plan $\{x=0\}$ est invariant pour la structure de Poisson $\Lambda$ (donc pour $\eta_{*} \Pi$ ).

Par un raisonnement similaire, on montre qu'il existe un changement de coordonnées $\delta$ de classe $C^{\infty}$ au voisinage de l'origine laissant fixe le plan $\{x=0\}$ et tel que $\{y=0\}$ soit invariant pour $(\delta \circ \eta)_{*} \Pi$. Enfin on réitère ce même raisonnement parallèlement à $\{x=0\}$ et $\{y=0\}$ pour obtenir le lemme.

Ainsi, pour le dernier modèle $\Pi=\Pi_{0}+\Pi_{\infty}$, il existe un difféomorphisme $\xi=\operatorname{id}_{\mathbb{R}^{3}}+\xi_{\infty}$, avec $\xi_{\infty}$ plat en 0 et tel que $\xi_{*} \Pi$ soit une structure de Poisson diagonale. Comme les réels $a, b$ et $c$ sont non nuls nous pouvons écrire :

$$
\bar{\Pi}=\xi_{*} \Pi=(1+\bar{h}) \Pi_{0},
$$

où $\bar{h}$ est plat à l'origine. Pour finir nous allons utiliser la méthode du chemin pour montrer que l'on peut éliminer le terme plat $\bar{h} \Pi_{0}$. Posons :

$$
\Pi_{t}=(1+t \bar{h}) \Pi_{0}=h_{t} \Pi_{0} .
$$

On cherche une famille $\left(\varphi_{t}\right)$ de difféomorphismes telle que $\left(\varphi_{t}\right)_{*} \Pi_{t}=\Pi_{0}$. Ce qui revient à chercher une famille $\left(Z_{t}\right)$ de champs de vecteurs vérifiant :

$$
\left[Z_{t}, \Pi_{t}\right]+\frac{\mathrm{d} \Pi_{t}}{\mathrm{~d} t}=0
$$

On doit donc résoudre l'équation :

$$
\left[Z_{t}, \Pi_{t}\right]=-\bar{h} \Pi_{0}
$$

Or, on sait que $\Pi_{0}$ se décompose en $\Pi_{0}=X_{0} \wedge Y_{0}$ où $X_{0}$ est le rotationnel de $\Pi_{0}$ relativement à la forme volume canonique $\Omega_{0}$. Cherchons $Z_{t}$ sous la forme $Z_{t}=g_{t} X_{0}$. L'équation (25) devient :

$$
\left[g_{t} X_{0}, h_{t} \Pi_{0}\right]=-\bar{h} \Pi_{0}
$$

Compte tenu du fait $\left[X_{0}, \Pi_{0}\right]=0$, il vient :

$$
-\bar{h} \Pi_{0}=\left(g_{t} X_{0}\left(h_{t}\right)-h_{t} X_{0}\left(g_{t}\right)\right) \Pi_{0}=-h_{t}^{2}\left(X_{0}\left(\frac{g_{t}}{h_{t}}\right)\right) \Pi_{0}
$$

TOME $125-1997-\mathrm{N}^{\circ} 4$ 
Il suffit de résoudre l'équation :

$$
X_{0}\left(\frac{g_{t}}{h_{t}}\right)=\frac{\bar{h}}{h_{t}^{2}} .
$$

Mais, par hypothèse $X_{0}$ est hyperbolique en 0 et $\bar{h}$ plate en 0 donc, d'après le théorème 3.2 , cette équation admet au moins une solution. Nous venons donc de montrer qu'il existe une famille $\left(\varphi_{t}\right)$ de difféomorphismes telle que

$$
\left(\varphi_{t}\right)_{*} \Pi_{t}=\Pi_{0} .
$$

On en déduit que la structure de Poisson $\Pi$ est $C^{\infty}$-isomorphe à $\Pi_{0}=f(\varrho) \Pi^{2}+\varrho^{q} \widetilde{\Pi}^{2}$, ce qui achève la démonstration du théorème 4.1.

REmarque. - Deux méthodes de résolution différentes ont été utilisées pour passer du formel au $C^{\infty}$. La première, qui repose essentiellement sur la proposition 3.1, est valable pour les cas A), B) et C); tandis que la seconde est efficace uniquement pour les cas A), C) et D).

\section{Singularités de 1-formes intégrables}

En dimension 3, à toute 1-forme intégrable est associée une structure de Poisson via l'isomorphisme défini par : $\omega \mapsto \Pi$ si et seulement si $i_{\Pi} \Omega_{0}=\omega$, où $\Omega_{0}$ désigne la forme volume canonique de $\mathbb{R}^{3}$. La condition d'intégrablité $\omega \wedge \mathrm{d} \omega=0$ est équivalente à $\Pi \wedge D_{\Omega_{0}}(\Pi)=0$.

Considérons deux 1-formes intégrables $\omega$ et $\omega^{\prime}$ qui sont $C^{\infty}$-conjuguées, c'est-à-dire qu'il existe un difféomorphisme $\varphi$ de classe $C^{\infty}$ tel que $\varphi^{*} \omega=\omega^{\prime}$. Notons $\Pi$ et $\Pi^{\prime}$ respectivement les structures de Poisson associées à $\omega$ et $\omega^{\prime}$. Nous avons

$$
\varphi^{*} \omega=\varphi^{*}\left(i_{\Pi} \Omega_{0}\right)=i_{\varphi_{*} \Pi} \varphi^{*} \Omega_{0}=(J \varphi) i_{\varphi_{*} \Pi} \Omega_{0} .
$$

Donc on a la relation $\Pi^{\prime}=(J \varphi) \varphi_{*} \Pi$ où $J \varphi$ est le Jacobien de $\varphi$. Cependant, les formes normales des 1-formes intégrables ne sont pas une transcription des formes normales des structures de Poisson. Voici un contre-exemple.

Contre-EXemple. - Soit $\omega_{2}$ la 1-forme intégrable donnée par :

$$
\omega_{2}=y z \mathrm{~d} x+m z x \mathrm{~d} y+n x y \mathrm{~d} z,
$$

avec $m, n \in \mathbb{N}^{*}$. Posons $\varrho(x, y, z)=x y^{m} z^{n}$ et considérons la 1-forme intégrable suivante :

$$
\omega=\left(1+\varrho^{q}\right) \omega_{2} .
$$


Nous allons montrer que $\omega$ est $C^{\infty}$-conjuguée à $\omega_{2}$. Pour ce faire, on considère le difféomorphisme :

$$
\varphi(x, y, z)=(x \theta(\varrho), y, z)
$$

Il s'agit de déterminer une fonction $\theta(\varrho)$ ne s'annulant pas à l'origine telle que $\varphi^{*} \omega_{2}=\omega$. Cette dernière relation est équivalente à :

$$
y z \mathrm{~d}(x \theta)+m z x \theta \mathrm{d} y+n x y \theta \mathrm{d} z=\left(1+\varrho^{q}\right) \omega_{2} .
$$

Ce qui peut encore s'écrire :

$$
\omega_{2} \theta+x y z \theta^{\prime} \mathrm{d} \varrho=\left(1+\varrho^{q}\right) \omega_{2} .
$$

En remarquant que

$$
\frac{\omega_{2}}{x y z}=\frac{d \varrho}{\varrho}
$$

il vient

$$
\theta \mathrm{d} \varrho+\varrho \theta^{\prime} \mathrm{d} \varrho=\left(1+\varrho^{q}\right) \mathrm{d} \varrho .
$$

On doit donc résoudre l'équation

$$
(\varrho \theta)^{\prime}=1+\varrho^{q} .
$$

On prend la solution donnée par :

$$
\theta(\varrho)=\frac{1}{\varrho} \int_{0}^{\varrho}\left(1+r^{q}\right) \mathrm{d} r .
$$

Ceci prouve que $\omega_{2}$ est $C^{\infty}$-conjuguée à $\omega$.

Pourtant, les structures de Poisson associées respectivement à $\omega_{2}$ et $\omega$ ne sont pas isomorphes. En effet, ces structures de Poisson sont

$$
\begin{aligned}
\Pi^{2} & =c x y \frac{\partial}{\partial x} \wedge \frac{\partial}{\partial y}+a y z \frac{\partial}{\partial y} \wedge \frac{\partial}{\partial z}+b z x \frac{\partial}{\partial z} \wedge \frac{\partial}{\partial x} \\
\Pi & =\left(1+\varrho^{q}\right) \Pi^{2}
\end{aligned}
$$

Nous avons vu dans le chapitre précédent que ces deux structures de Poisson ne sont pas isomorphes.

Dans [Ce-LN], on donne des résultats de formes normales de 1-formes intégrables sur $\mathbb{R}^{n}$. En se servant de l'étude faite sur les structures de Poisson, nous précisons ces résultats dans le cas où $n=3$. Partons d'une 
forme intégrable $\omega$ de classe $C^{\infty}$ au voisinage de $O \in \mathbb{R}^{3}$ ayant pour 2-jet en 0

$$
\omega_{2}=a y z \mathrm{~d} x+b z x \mathrm{~d} y+c x y \mathrm{~d} z,
$$

avec $(a-b)(b-c)(c-a) \neq 0$. La structure de Poisson $\Pi$ associée à $\omega$ est définie par la relation $\omega=i_{\Pi} \Omega_{0}$. Supposons que si l'un des coefficients $a, b$ ou $c$ est nul alors les deux autres sont de signe contraire.

Nous savons que sous ces hypothèses la structure de Poisson $\Pi$ est isomorphe en classe $C^{\infty}$ à l'un des quatre modèles locaux du théorème 2.1. En d'autres termes, il existe un changement de coordonnées $\varphi$ de classe $C^{\infty}$ au voisinage de l'origine tel que $\varphi^{*} \omega=(J \varphi) \omega_{0}$, où $J \varphi$ est le Jacobien de $\varphi$ et $\omega_{0}$ l'une des formes intégrables suivantes

$$
\begin{aligned}
& \mathcal{T}_{0}=\omega_{2}, \\
& \mathcal{T}_{1}=\omega_{2}+y^{m_{1}} z^{m_{2}}(\alpha y \mathrm{~d} z-z \mathrm{~d} y), \\
& \mathcal{T}_{2}=\left(1+\varrho^{q}\right) \omega_{2}, \\
& \mathcal{T}_{3}=\left(1+\alpha_{1} \varrho+\cdots+\alpha_{q-1} \varrho^{q-1}\right) \omega_{2}+\varrho^{q} \widetilde{\omega}_{2} .
\end{aligned}
$$

avec les notations du paragraphe précédent et $\widetilde{\omega}_{2}=\tilde{a} y z \mathrm{~d} x+\tilde{b} z x \mathrm{~d} y+$ $\tilde{c} x y \mathrm{~d} z$, le vecteur $(\tilde{a}, \tilde{b}, \tilde{c})$ n'étant pas colinéaire à $(a, b, c)$.

En fait, on peut même prendre $\varphi$ tel que sa linéarisée en 0 coïncide avec l'identité. On a donc $\omega=(1+h) \omega_{0}$, avec $h(0)=0$. Montrons qu'il est possible de se débarrasser de la fonction $h$.

Lemme 5.1. - La 1-forme intégrable $\omega$ est $C^{\infty}$-conjuguée à $\omega_{0}$.

Preuve. - Elle utilise la méthode du chemin. Posons $\omega_{t}=(1+t h) \omega_{0}$. Cherchons une famille de difféomorphismes $\left(\varphi_{t}\right)_{t}$ telle que $\left(\varphi_{t}\right)^{*} \omega_{t}=\omega_{0}$, pour tout $t$. Cela revient à chercher une famille de champs de vecteurs $\left(X_{t}\right)_{t}$ vérifiant :

$$
L_{X_{t}} \omega_{t}+\frac{\mathrm{d} \omega_{t}}{\mathrm{~d} t}=0
$$

où $L_{X_{t}}$ est la dérivation de Lie suivant le champ de vecteurs $X_{t}$. Cette équation (26) équivaut à :

$$
(1+t h) L_{X_{t}} \omega_{0}+t X_{t}(h) \omega_{0}=-h \omega_{0}
$$

Nous savons que chacun des quatre modèles locaux de structures de Poisson se décompose sous la forme

$$
\Pi_{0}=D_{\Omega_{0}}\left(\Pi_{0}\right) \wedge Y_{0}
$$


Fixons un tel champ de vecteurs $Y_{0}$ et posons

$$
X_{t}=f_{t} Y_{0}
$$

Il s'agit donc de déterminer une famille $\left(f_{t}\right)$ de fonctions différentiables vérifiant :

$$
(1+t h) L_{f_{t} Y_{0}} \omega_{0}+t f_{t} Y_{0}(h) \omega_{0}=-h \omega_{0} .
$$

Or, il découle de la relation $\omega_{0}=i_{\Pi_{0}} \Omega_{0}$ que l'on a

$$
i_{\left(f_{t} Y_{0}\right)} \omega_{0}=f_{t} i_{Y_{0}} \omega_{0}=0 \text {. }
$$

Par conséquent, on a

$$
L_{f_{t} Y_{0}} \omega_{0}=i_{\left(f_{t} Y_{0}\right)} \mathrm{d} \omega_{0}=f_{t} i_{Y_{0}} \mathrm{~d} \omega_{0}=f_{t} i_{Y_{0}} \mathrm{~d} i_{\Pi_{0}} \Omega_{0} .
$$

Mais par définition de l'opérateur $D_{\Omega_{0}}$ on a

$$
\mathrm{d} i_{\Pi_{0}} \omega_{0}=i_{D_{\Omega_{0}}\left(\Pi_{0}\right)} \omega_{0} \text {. }
$$

On trouve

$$
L_{f_{t} Y_{0}} \omega_{0}=f_{t} i_{Y_{0}} i_{D_{\Omega_{0}}\left(\Pi_{0}\right)} \Omega_{0}=f_{t} i_{\Pi_{0}} \Omega_{0}=f_{t} \omega_{0} .
$$

En reportant ceci dans (27) on obtient :

$$
\left((1+t h) f_{t}+t f_{t} Y_{0}(h)\right) \omega_{0}=-h \omega_{0} .
$$

Il suffit de prendre

$$
f_{t}=-\frac{h}{1+t\left(h+Y_{0}(h)\right)} .
$$

Ce qui montre la forme intégrable $\omega$ est conjuguée à $\omega_{0}$. D'où le lemme.

Il découle du lemme précédent que toute 1-forme intégrable ayant $\omega_{2}$ pour 2-jet en 0 est $C^{\infty}$-conjuguée à l'un des quatre types de 1 -formes $\mathcal{T}_{0}$, $\mathcal{T}_{1}, \mathcal{T}_{2}$ ou $\mathcal{T}_{3}$ ci-dessus. Nous allons voir qu'il y a exactement trois modèles locaux de 1 -formes intégrables.

Lemme 5.2. - Avec les notations précédentes, la 1-forme intégrable donnée par $\omega_{0}=\left(1+\varrho^{q}\right) \omega_{2}$ est $C^{\infty}$-conjuguée à $\omega_{2}$.

La preuve est faite dans le contre-exemple donné au début de ce paragraphe.

REMARque. - On peut aussi démontrer le lemme 5.2 en utilisant le fait que la fonction polynomiale $\varrho(x, y, z)$ est une intégrale première formelle faible au sens de $[\mathrm{M}-\mathrm{M}]$ ) (cela signifie que $\omega_{0} \wedge \mathrm{d} \varrho=0$ ). Il suffit alors d'appliquer [Ce-LN] qui dit ceci : lorsque $(a, b, c)$ est colinéaire à un triplet de $\mathbb{N}^{3}$ avec $(a-b)(b-c)(c-a) \neq 0$, pour que $\omega_{0}$ soit $C^{\infty}$-conjuguée à $\omega_{2}$ il faut et il suffit qu'elle admette une intégrale première formelle faible.

TOME $125-1997-\mathrm{N}^{\circ} 4$ 
Lemme 5.3. - Avec les notations précédentes la 1-forme

$$
\omega_{0}=\left(1+\alpha_{1} \varrho+\cdots+\alpha_{q-1} \varrho^{q-1}\right) \omega_{2}+\varrho^{q} \widetilde{\omega}_{2},
$$

est $C^{\infty}$-conjuguée à $\omega_{2}+\varrho^{q} \widetilde{\omega}_{2}$.

Preuve. - Posons

$$
\begin{aligned}
f(\varrho) & =1+\alpha_{1} \varrho+\cdots+\alpha_{q-1} \varrho^{q-1}, \\
\widetilde{\omega}_{2} & =\tilde{a} y z \mathrm{~d} x+\tilde{b} z x \mathrm{~d} y+\tilde{c} y x \mathrm{~d} z .
\end{aligned}
$$

Considérons le changement de coordonnées définies au voisinage de l'origine par $\varphi:(x, y, z) \mapsto(x \theta(\varrho), y \gamma(\varrho), z)$ avec $\theta \gamma=1$. La relation $\varphi^{*} \omega_{0}=\omega_{2}+\varrho^{q} \widetilde{\omega}_{2}$ se traduit par :

$$
\begin{aligned}
f(\varrho) \omega_{2}+x y z f(\varrho)(a \gamma d \theta+ & b \theta d \gamma)+\varrho^{q} \widetilde{\omega}_{2} \\
& +x y z \varrho^{q}(\tilde{a} \gamma \mathrm{d} \theta+\tilde{b} \theta \mathrm{d} \gamma)=\omega_{2}+\varrho^{q} \widetilde{\omega}_{2} .
\end{aligned}
$$

En utilisant les relations

$$
\frac{\omega_{2}}{x y z}=\frac{d \varrho}{\varrho}, \quad \theta \gamma=1,
$$

l'égalité précédente donne :

$$
f(\varrho)\left(a \frac{\theta^{\prime}}{\theta}+b \frac{\gamma^{\prime}}{\gamma}\right)+\varrho^{q}\left(\tilde{a} \frac{\theta^{\prime}}{\theta}+\tilde{b} \frac{\gamma^{\prime}}{\gamma}\right)=\frac{1-f(\varrho)}{\varrho} .
$$

Or, la relation $\theta \gamma=1$ implique

$$
\frac{\theta^{\prime}}{\theta}=-\frac{\gamma^{\prime}}{\gamma}
$$

Donc, il vient

$$
\frac{\theta^{\prime}}{\theta}\left((a-b) f(\varrho)+(\tilde{a}-\tilde{b}) \varrho^{q}\right)=\frac{1-f(\varrho)}{\varrho} .
$$

On sait résoudre cette équation, par conséquent il existe bien un changement de coordonnées $\varphi$ de classe $C^{\infty}$ tel que $\varphi^{*} \omega_{0}=\omega_{2}+\varrho^{q} \widetilde{\omega}_{2}$.

En définitive, nous avons démontré le théorème suivant : BULLETIN DE LA SOCIÉTÉ MATHÉMATIQUE DE FRANCE 
ThÉORÈme 5.1. - Soit $\omega$ une 1-forme intégrable de classe $C^{\infty}$ au voisinage de l'origine $O \in \mathbb{R}^{3}$ ayant pour 2 -jet en 0

$$
\omega_{2}=a y z \mathrm{~d} x+b z x \mathrm{~d} y+c x y \mathrm{~d} z,
$$

avec $(a-b)(b-c)(c-a) \neq 0$. Supposons que si l'un des coefficients $a, b$ ou $c$ est nul, les deux autres sont de signe contraire. Alors $\omega$ est $C^{\infty}$-conjuguée à l'un des modèles suivants:

$$
\begin{aligned}
& \mathcal{F}_{0}=\omega_{2}, \\
& \mathcal{F}_{1}=\omega_{2}+y^{m_{1}} z^{m_{2}}(\alpha y \mathrm{~d} z-z \mathrm{~d} y), \\
& \mathcal{F}_{2}=\omega_{2}+\varrho^{q} \widetilde{\omega}_{2},
\end{aligned}
$$

ò̀ $\widetilde{\omega}_{2}=\tilde{a} y z \mathrm{~d} x+\tilde{b} z x \mathrm{~d} y+\tilde{c} x y \mathrm{~d} z$, le vecteur $(\tilde{a}, \tilde{b}, \tilde{c})$ n'étant pas colinéaire $\grave{a}(a, b, c)$.

Remarque. - Nous avons vu au paragraphe IV que pour $j=0$ ou 1 , la structure de Poisson définie par

$$
i_{\Pi_{j}} \mathrm{~d} x \wedge \mathrm{d} y \wedge \mathrm{d} z=\mathcal{F}_{j}
$$

se décompose en $\Pi_{j}=X_{j} \wedge Y_{j}$, où $X_{j}$ est le rotationnel de $\Pi_{j}$ et $Y_{j}$ est un champ de vecteurs qui commute avec $X_{j}$.

De plus, $X_{j}$ et $Y_{j}$ sont polynomiaux (ces champs de vecteurs ont été déterminés dans la section précédente). Cela est également valable pour le modèle $\mathcal{F}_{2}$ si on suppose que les vecteurs $(a, b, c),(\tilde{a}, \tilde{b}, \tilde{c})$ et $(1,1,1)$ sont linéairement indépendants. On peut donc conclure que $\mathcal{F}_{0}$ et $\mathcal{F}_{1}$ sont tangentes à une action commutative polynomiale tandis que $\mathcal{F}_{2}$ l'est génériquement.

\section{BIBLIOGRAPHIE}

[A] Arnol'd (V.-I.). - Geometrical methods in the theory of differential equations. - Grundlehren der mathimatischen, Springer Verlag, second ed., 1988.

[Ca-LN] Camacho (C.), Lins Neto (A.). - The topology of integrable differential forms near a singularity, Pub. Math. I.H.E.S., t. 55, 1982, p. 5-35. 
[Ce-LN] Cerveau (D.), Lins Neto (A.). - Formes tangentes à des actions commutatives, Ann. Fac. Sci. Toulouse, t. VI, 1984, p. 51-85.

[Ch] Chaperon (M.).— Géométrie différentielle et singularités de systèmes dynamiques, Astérisque, 1986, p. 138-139.

[C1] Conn (J.-F.). - Normal forms for analytic Poisson structures, Ann. of Math., t. 119 (2), 1984, p. 576-601.

[C2] Conn (J.-F.). - Normal forms for smooth Poisson structures, Ann. of Math., t. 121 (2), 1985, p. 565-593.

[De] Desolneux Moulis (N.). - Linéarisation de certaines structures de Poisson, Pub. Dép. Math., Université Claude Bernard Lyon, 1986.

[D1] Dufour (J.-P.). - Linéarisation de structures de Poisson, J. Diff. Geometry, t. 32, 1990, p. 415-428.

[D2] Dufour (J.-P.). - Quadratisation de structures de Poisson, Preprint, Montpellier, 1993 .

[D-H] Dufour (J.-P.), Haraki (A.). - Rotationnels et structures de Poisson quadratiques, C.R. Acad. Sci. Paris, t. 312, I, 1991, p. 137-140.

[D-M] Dufour (J.-P.), Molinier (J.-C.). - Une nouvelle famille de d'algèbre de Lie non dégénérées, Indag. Math., N.S., t. 6 (1), 1995, p. $67-82$.

[D-W] Dufour (J.-P.), Wade (A.). - Formes normales de structures de Poisson ayant un 1-jet nul, à paraître dans J. of Geometry and Phys.

[H] Haraki (A.). - Structures de Poisson quadratiques, Thèse, Montpellier, 1993 .

[K] Koszul (J.-L.). - Crochets de Schouten-Nijenhuis et cohomologie, Astérisque, t. 127 bis (hors série), 1985, p. 257-271.

[L-M] Libermann (P.), MARLE (M.). - Symplectic geometry and analytical mechanics. - Reidel, Dordrecht, Holland, 1987.

[L] Lichnerowicz (A.). - Les variétés de Poisson et leurs algèbres de Lie associées, J. Diff. Geometry, t. 12, 1977, p. 253-300.

[L-N] Lins Neto (A.). - Local structural stablity of $C^{2}$-intégrable 1-forms, Ann. Inst. Fourier, Grenoble, t. 27 (2), 1977, p. 197-225.

[Ma] Martinet (J.).- Normalisation des champs de vecteurs holomorphes (d'après A.D. Brujno), Séminaire Bourbaki, t. 564, 1980-1981.

[M-M] Mattei (J.-F.), Moussu (R.). - Holonomie et intégrales premières, Ann. Sci. École Normale Supérieure, t. 13, 198o, p. 469-523.

[Mo] Moliner (J.-C.). - Linéarisation de structures de Poisson, Thèse, Montpellier, 1993.

[R] Roussarie (R.). - Modèles locaux de champs de vecteurs et formes, Astérisque, 1975 . 
[Sch] Schouten (J.-A.). - On the differential operators of first order in tensor calculus, Convegno Int. Geom. Diff. Italia, 1953, p. 1-7, Roma, Cremonese.

[Wa1] Wade (A.). - Normalisation formelle de structures de Poisson, C.R. Acad. Sci. Paris, t. 324 I, 1997, p. 531-536.

[Wa2] Wade (A.). - Normalisation de structures de Poisson, Thèse, Montpellier, 1996.

[We1] Weinstein (A.). - The local structure of Poisson manifold, J. Diff. Geometry, t. 18, 1983, p. 523-557.

[We2] Weinstein (A.). - The Modular Automorphism Group of a Poisson Manifold, Preprint (mai 1996). 